Marquette University

e-Publications@Marquette

Biomedical Engineering Faculty Research and

Publications

Biomedical Engineering, Department of

8-21-2013

Few-view Single Photon Emission Computed Tomography (SPECT) Reconstruction Based on a Blurred Piecewise Constant Object Model

Paul Arthur Wolf

Marquette University, paul.wolf@marquette.edu

Jakob S. Jørgensen

Technical University of Denmark

Taly Gilat Schmidt

Marquette University, tal.gilat-schmidt@marquette.edu

Emil Y. Sidky

University of Chicago

Accepted version. Physics in Medicine \& Biology, Vol. 58, No. 16 (August 21, 2013): 5629-5652. DOI.

(C) 2013 Institute of Physics. Used with permission. 


\title{
Few-View Single Photon Emission Computed Tomography (SPECT) Reconstruction Based On A Blurred Piecewise Constant Object Model
}

\author{
Paul A. Wolf ${ }^{1}$ \\ Department of Biomedical Engineering, Marquette University \\ Milwaukee, WI \\ Jakob S. Jørgensen² \\ Department of Applied Mathematics and Computer Science, \\ Technical University of Denmark \\ Lyngby, Denmark \\ Taly Gilat Schmidt ${ }^{1}$ \\ Department of Biomedical Engineering, Marquette University \\ Milwaukee, WI \\ Emil Y. Sidky 3 \\ Department of Radiology, University of Chicago, \\ Chicago, IL
}

\begin{abstract}
A sparsity-exploiting algorithm intended for few-view Single Photon Emission Computed Tomography (SPECT) reconstruction is proposed and characterized. The algorithm models the object as piecewise constant subject to a blurring operation. To validate that the algorithm closely approximates the true object in the noiseless case, projection data were generated from an object assuming this model and using the system matrix. Monte Carlo
\end{abstract}

Physics in Medicine and Biology, Vol. 58, No. 16 (August 2013): pg. 5629-5652. DOI. This article is @ Institute of Physics and permission has been granted for this version to appear in e-Publications@Marquette. Institute of Physics does not grant permission for this article to be further copied/distributed or hosted elsewhere without the express permission from Institute of Physics. 
NOT THE PUBLISHED VERSION; this is the author's final, peer-reviewed manuscript. The published version may be accessed by following the link in the citation at the bottom of the page.

simulations were performed to provide more realistic data of a phantom with varying smoothness across the field of view and a cardiac phantom.

Reconstructions were performed across a sweep of two primary design parameters. The results demonstrate that the algorithm recovers the object in a noiseless simulation case. While the algorithm assumes a specific blurring model, the results suggest that the algorithm may provide high reconstruction accuracy even when the object does not match the assumed blurring model. Generally, increased values of the blurring parameter and Total Variation (TV) weighting parameters reduced streaking artifacts, while decreasing spatial resolution. The proposed algorithm demonstrated higher correlation with respect to the true phantom compared to Maximum Likelihood Expectation Maximization (MLEM) reconstructions. Images reconstructed with the proposed algorithm demonstrated reduced streaking artifacts when reconstructing from few views compared to MLEM. The proposed algorithm introduced patchy artifacts in some reconstructed images, depending on the noise level and the selected algorithm parameters. Overall, the results demonstrate preliminary feasibility of a sparsity-exploiting reconstruction algorithm which may be beneficial for few-view SPECT.

Keywords: sparsity-exploiting reconstruction, SPECT, tomographic reconstruction

\section{Introduction}

Single Photon Emission Computed Tomography (SPECT) provides noninvasive images of the distribution of radiotracer molecules. Dynamic SPECT provides information about tracer uptake and washout from a series of time-sequence images. Dynamic SPECT acquisition methods measuring time activity curves on the order of minutes have been developed (Gullberg et al 2010, Gullberg 2004). However, the dynamic uptake and washout of some tracers occurs over a period of just several seconds, requiring better temporal sampling. Stationary ring-like multi-camera systems are being developed to provide rapid dynamic acquisitions with high temporal sampling (Beekman et al 2005, Furenlid et al 2004, Beekman and Vastenhouw 2004). Reducing the number of cameras reduces the cost of such systems but also reduces the number of views acquired, limiting the angular sampling of the system. Novel few-view image reconstruction methods may be beneficial and are being investigated for the application of dynamic SPECT (Ma et al 2013).

Physics in Medicine and Biology, Vol. 58, No. 16 (August 2013): pg. 5629-5652. DOI. This article is (C) Institute of Physics and permission has been granted for this version to appear in e-Publications@Marquette. Institute of Physics does not grant permission for this article to be further copied/distributed or hosted elsewhere without the express permission from Institute of Physics. 
The feasibility of reconstructing from angularly undersampled, or few-view data, has recently been explored for CT (Sidky and Pan 2008, Chen et al 2008, Duan et al 2009, Ritschl et al 2011, Sidky et al 2006). These investigations are based on exploitation of gradientmagnitude sparsity, an idea promoted and theoretically investigated in the field of Compressed Sensing (CS). Few-view, sparsity-exploiting CT reconstruction algorithms promote gradient-magnitude sparsity by minimizing image total variation (TV). Success of these algorithms in allowing sampling reduction follows from an object model which is approximately piecewise constant, a model that may not apply well for SPECT objects. A previous study indicated potential noise and artifact issues when implementing TV minimization for few-view SPECT reconstruction (Hsieh 2010). The SPECT object function quantifies the physiological uptake of a radiolabelled tracer in the body. In some applications, the transition between different uptake regions in the SPECT object is expected to be smoother than the transition between $X$-ray attenuation coefficients in the CT object. One study proposed exploiting sparsity in the curvelet domain for SPECT imaging (Mukherjee 2013). The goal of this work is to modify the idea of exploiting gradient-magnitude sparsity to allow for smoother transitions between regions of approximately constant values of tracer concentration.

This paper proposes an iterative algorithm for few-view SPECT reconstruction that allows for smoothed step-like variation within the object by phenomenologically modeling the SPECT object as a blurred version of a piecewise constant object. Using this model, a first-order primal-dual technique is implemented as an iterative procedure (Chambolle and Pock 2011, Sidky et al 2012). The purpose of this study was to characterize the performance of the algorithm under varying sampling and noise conditions, including cases where the object does not match the phenomenological model. Images reconstructed by Maximum-Likelihood Expectation Maximization (MLEM) serve as a reference. This study focuses on small-animal pinhole SPECT, where stationary systems with a limited number of cameras have been proposed for dynamic imaging (Beekman et al 2005, Furenlid et al 2004, Beekman and Vastenhouw 2004). The article is organized as follows: Section 2 provides the image reconstruction theory and algorithm. Section 3 demonstates the algorithm with data generated using the system matrix. Sections 4 and

Physics in Medicine and Biology, Vol. 58, No. 16 (August 2013): pg. 5629-5652. DOI. This article is (C) Institute of Physics and permission has been granted for this version to appear in e-Publications@Marquette. Institute of Physics does not grant permission for this article to be further copied/distributed or hosted elsewhere without the express permission from Institute of Physics. 
5 demonstrate the algorithm with data generated from a realistic Monte Carlo simulation of a SPECT system. Section 6 summarizes the results.

\section{The algorithm}

The iterative image reconstruction algorithm (IIR) is designed by defining an optimization problem which implicitly specifies the object function based on a realistic data model and a model for object sparsity. In this preliminary investigation, the specified optimization problem is solved in order to characterize its solution and the solution's appropriateness for few-view/dynamic SPECT imaging. Future work will consider algorithm efficiency by designing IIR for approximate solution of the proposed optimization problem.

\subsection{The SPECT optimization problem}

The proposed SPECT optimization problem is formulated as an unconstrained minimization of an objective function which is the sum of a data fidelity term and an image regularity penalty. The design of both terms expresses the proper SPECT noise model and a modified version of gradient-magnitude object sparsity. We first describe how standard gradient-magnitude sparsity is incorporated into a SPECT optimization problem, and then we present our modified optimization which accounts for the smoother variations expected in a SPECT object function.

\subsubsection{Unconstrained minimization for gradient- magnitude sparsity exploiting SPECT IIR}

In expressing the SPECT data fidelity term, the data are modeled as a Poisson process the mean of which is described by the following linear system of equations:

$$
\boldsymbol{g}=\boldsymbol{H} \boldsymbol{f}
$$

where $\boldsymbol{H}$ is the system matrix that describes the probability that a photon emitted from a certain location in the object vector, $\boldsymbol{f}$,

Physics in Medicine and Biology, Vol. 58, No. 16 (August 2013): pg. 5629-5652. DOI. This article is @ Institute of Physics and permission has been granted for this version to appear in e-Publications@Marquette. Institute of Physics does not grant permission for this article to be further copied/distributed or hosted elsewhere without the express permission from Institute of Physics. 
contributes to the measured data vector, $\boldsymbol{g}$, at a certain location. Iterative tomographic image reconstruction techniques such as MLEM and Ordered Subset Expectation Maximization (OSEM) maximize the log-likelihood of this Poisson random variable (Shepp and Vardi 1982, Hudson and Larkin 1994, Vandenberghe et al 2001). This is equivalent to minimizing the Kullback-Leibler $(\mathrm{KL})$ data divergence $\left(D_{\mathrm{KL}}\right)$ (Barrett and Myers 2004). For the present application of few-view SPECT, the data are acquired over too few views to provide a unique maximum likelihood image. In the limit of infinite photon counts and assuming that the mean model in (1) perfectly describes the imaging system, the underlying object function still cannot be determined because (1) is underdetermined.

In order to arrive at a reasonable solution, additional information or assumptions on the object function are needed. Recently, exploitation of gradient-magnitude sparsity has received much attention and has been implemented in IIR for few-view CT (Chen et al 2008, Sidky et al 2009). This idea is an example of a general strategy under much recent investigation in CS, where sampling conditions are based on some form of identified sparsity in the image. In our application the strategy calls for narrowing the solution space to only images that exactly solve our linear model in (1). Among those images, the solution with the lowest TV is sought. In practice, this solution can be obtained approximately by combining a data fidelity term with a TV penalty, where the combination coefficient in front of the TV penalty is vanishingly small. The TV- $D_{\mathrm{KL}}$ sum yields the following minimization:$$
\operatorname{minimize}_{\{}\left\{D_{K L}(\boldsymbol{g}, \boldsymbol{H} \boldsymbol{f})+\gamma\|(|\boldsymbol{D f}|)\| 1\right\},
$$

where $\boldsymbol{D}$ is a discrete gradient operator and $\mathrm{Y}$ is a weighting parameter. The first addend of the objective function is the $\mathrm{KL}$ divergence between the measured data, $\boldsymbol{g}$, and the forward projection of the object estimate, $\boldsymbol{H} \boldsymbol{f}$. The term $\boldsymbol{D} \boldsymbol{f}$ is a vector valued image with the spatial image gradient vector at each element. The gradient magnitude image of $\boldsymbol{D} \boldsymbol{f}$ is represented by $|\boldsymbol{D} \boldsymbol{f}|$, which consists of the magnitude of the gradient vectors at each element. The $/ 1$-norm corresponds to summing all of the elements of the gradient magnitude 
image. So, the second addend represents the TV of $\boldsymbol{f}$, weighted by $Y$. For sparsity-exploiting IIR, $\mathrm{Y}$ is chosen so that the data fidelity term far outweighs the TV-term. The role of the TV term is simply to break the degeneracy in the objective function among all solutions of (1).

The success of TV minimization for few-view CT IIR relies on the assumption that the $X$-ray attenuation coefficient map is approximately piecewise constant. Directly promoting sparsity of the gradient-magnitude image may not be as beneficial for SPECT, as in some cases tracer uptake may vary smoothly within objects, and borders of objects may show a smoothed step-like dependence. For example, some regions of the heart are supplied by a single coronary artery while other regions are supplied by multiple coronary arteries (Donato et al 2012, Pereztol-Valdés et al 2005). Thus, cardiac perfusion studies may be one application for which the blurred piecewise constant model is appropriate. As another example, tumor vascularization is heterogeneous, with vascularization often varying from the tumor center to the periphery (Jain 1988). Therefore, our goal here is to find a sparsity-exploiting formulation which allows some degree of smoothness between regions with different uptake.

\subsubsection{Unconstrained minimization for sparsity exploiting IIR using a blurred piecewise constant object model}

In this work the TV minimization detailed in (2) is modified to allow for rapid but smooth variation by phenomenologically modeling objects as piecewise constant subject to a shift-invariant blurring operation.

The additional blurring operation can be incorporated into the framework developed above by minimizing the weighted sum of the TV of an intermediate piecewise constant object estimate and $D_{\mathrm{KL}}$ between the measured data and the projection data of the blurred object estimate. The modified TV-minimization objective is:

$$
\operatorname{minimize}_{f}\left\{D_{\mathrm{KL}}(\boldsymbol{g}, \boldsymbol{H u})+\gamma\|(|\boldsymbol{D} \boldsymbol{f}|)\|_{1}\right\},
$$

Physics in Medicine and Biology, Vol. 58, No. 16 (August 2013): pg. 5629-5652. DOI. This article is @ Institute of Physics and permission has been granted for this version to appear in e-Publications@Marquette. Institute of Physics does not grant permission for this article to be further copied/distributed or hosted elsewhere without the express permission from Institute of Physics. 
where $\boldsymbol{u}$ is the object estimate and $\boldsymbol{f}$ is an intermediate image with sparse gradient-magnitude. Eq. 3 is similar to Eq. 2 except that the $D_{\mathrm{KL}}$ term is calculated using $\boldsymbol{u}$, which is a blurred version of the image $\boldsymbol{f}$ used in TV minimization. These object representations are related by $\boldsymbol{u}=\boldsymbol{M G M \boldsymbol { f }}$, where $\boldsymbol{M}$ is a support preserving image mask and $\boldsymbol{G}$ is a Gaussian blurring operation with standard deviation $r$. The operators $\boldsymbol{M}$ and $\boldsymbol{G}$ are symmetric so $\boldsymbol{M}^{\top}=\boldsymbol{M}$ and $\boldsymbol{G}^{\top}=\boldsymbol{G}$ The operator $\boldsymbol{G}$ extends data outside the physical support of the system assumed by $\boldsymbol{H}$ so the image mask $\boldsymbol{M}$ must be applied before and after $\boldsymbol{G}$. This optimization problem has two design parameters, $Y$, which is the weighting of the TV term, and $r$, which is the standard deviation of the Gaussian blurring kernel. The blurring parameter, $r$, represents smoothness in the underlying object, as opposed to blurring introduced by the imaging system. When $r=0$, this formulation defaults to TV minimization problem in (2). If $\gamma=0$, the formulation described by (3) minimizes $D_{\mathrm{KL}}$, which is implicitly minimized in MLEM. The final image estimate is $\boldsymbol{u}$, the result of blurring and masking the intermediate piecewise constant object, $\boldsymbol{f}$. Minimizing (3) jointly enforces sparsity (by requiring a low TV of $\boldsymbol{f}$ ) and encourages data match (by requiring a low $\left.D_{\mathrm{KL}}\right)$.

\subsection{Optimization algorithm}

Only recently have algorithms been developed that can be applied to large-scale, non-smooth convex optimization problems such as that posed by (3). Sidky et al (2012) adapts the Chambolle-Pock (CP) algorithm to solve the TV- $D_{\mathrm{KL}}$ sum described by (2) (Chambolle and Pock 2011). Applying the model as described above, this prototype can be modified to solve the optimization posed by (3). Pseudo-code describing this algorithm is written below.

This algorithm is a modification of Algorithm 5 described in previous work by Sidky et al (2012). The convergence criterion described in that work was used here.

Simulation studies were conducted to characterize the performance of the proposed reconstruction technique over a range of angular sampling conditions, including cases in which the object does not match the phenomenological blurred piecewise constant model. The first simulation study used noiseless data generated from the

Physics in Medicine and Biology, Vol. 58, No. 16 (August 2013): pg. 5629-5652. DOI. This article is (C) Institute of Physics and permission has been granted for this version to appear in e-Publications@Marquette. Institute of Physics does not grant permission for this article to be further copied/distributed or hosted elsewhere without the express permission from Institute of Physics. 
system forward model to validate that the reconstruction technique closely approximates the true object when the correct blurring and system models are used, and to investigate the effects of the design parameters $r$ and $\mathrm{y}$. Another study reconstructed data generated by Monte Carlo simulation for a range of sampling and noise conditions and for varying values of algorithm parameters $r$ and $\gamma$.

\section{Inverse crime simulation study}

This study was designed to validate that the reconstruction technique approximates the true object when both the object model and system model are known exactly. The simulated object was generated from the object model ( $\boldsymbol{u}=\mathbf{M G M f}$, for a specified $\boldsymbol{f}$ ) and data were generated using the system forward model ( $\boldsymbol{g}=\boldsymbol{H u}$ ), where $\boldsymbol{H}, \boldsymbol{G}$, and $\boldsymbol{M}$ are the matrices used by the reconstruction algorithm. Cases such as this, in which the data were produced directly from the model are referred to as the "inverse crime" (Kaipio and Somersalo 2005). This is investigated in the many-view (128 views) and few-view ( 9 views) cases. We also examined the effects of different blurring models on the gradient-magnitude sparsity of the intermediate object $\boldsymbol{f}$. The algorithm could enable further reductions in sampling if the blurring model increases the gradient-magnitude sparsity compared to the conventional TV minimization term. In order to investigate the performance of the reconstruction with inconsistent data, Poisson noise was added to the data and the study was repeated. We refer to this as the "noisy" case.

\subsection{Methods}

\subsubsection{Phantom}

The intermediate piecewise constant object, $\boldsymbol{f}_{\text {true, }}$ was defined on a $128 \times 128$ grid of $0.55-\mathrm{mm} \times 0.55-\mathrm{mm}$ pixels, representing a $6-\mathrm{mm}$ diameter disk embedded in a 38-mm diameter disk. The intensity of the small disk was 2000 arbitrary units and the intensity of the large disk was 200 arbitrary units. A Gaussian blurring kernel with standard deviation, $r_{\text {true }}=0.75$ pixels was applied to this intermediate object, $\boldsymbol{f}_{\text {true, }}$ to generate the ground-truth object, $\boldsymbol{u}_{\text {true, }}$ which was used as the phantom. The intermediate piecewise constant object, $\boldsymbol{f}_{\text {true, }}$ and the

Physics in Medicine and Biology, Vol. 58, No. 16 (August 2013): pg. 5629-5652. DOI. This article is @ Institute of Physics and permission has been granted for this version to appear in e-Publications@Marquette. Institute of Physics does not grant permission for this article to be further copied/distributed or hosted elsewhere without the express permission from Institute of Physics. 
ground-truth object, $\boldsymbol{u}_{\text {true, }}$ are shown in figure 1 . The ground-truth object was used in the inverse crime simulations.
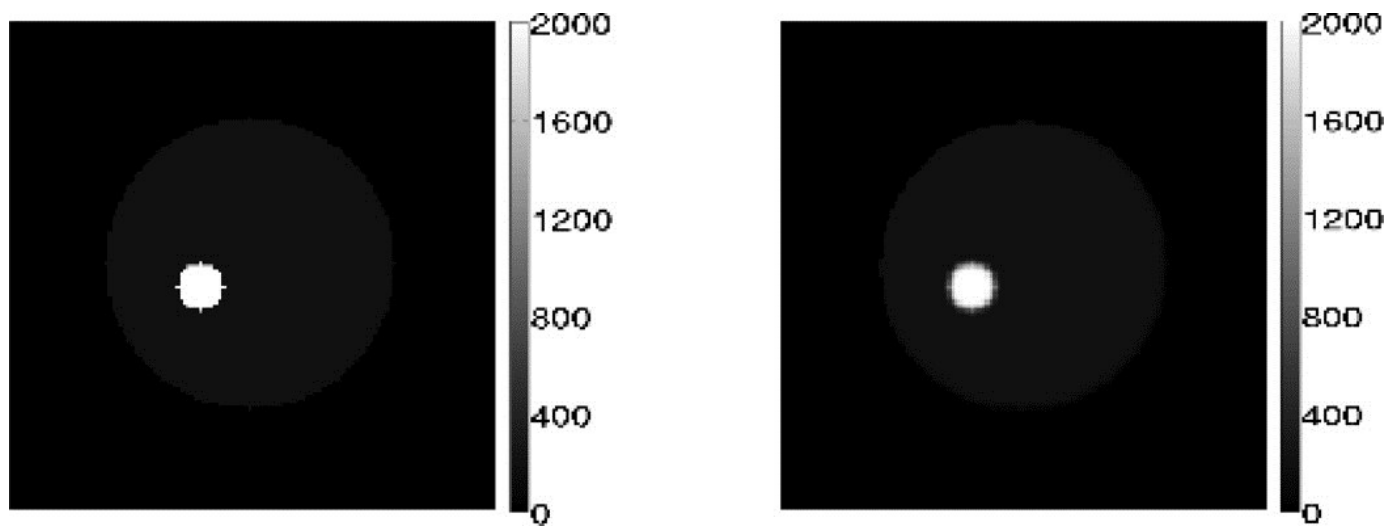

Figure 1.

Intermediate piecewise constant object, $\boldsymbol{f}_{\text {true, }}$ (left) and the ground-truth object, $\boldsymbol{u}_{\text {true, }}$ obtained by blurring $\boldsymbol{f}_{\text {true }}$ with $r_{\text {true }}=0.75$ pixels (right). $\boldsymbol{u}_{\text {true }}$ was the phantom used in the inverse crime simulations.

\subsubsection{Simulation}

Projection data of the pixelized ground-truth object was generated from the system matrix. The system matrix was estimated using Siddon's raytracing algorithm for a single-pinhole SPECT system with 128 discrete $1 \mathrm{~mm}$ detector bins, $3 \mathrm{~mm}$ pinhole diameter, $1.0 \mathrm{~mm}$ FWHM intrinsic detector resolution, $35 \mathrm{~mm}$ pinhole-to-object distance and $63.5 \mathrm{~mm}$ pinhole-to-detector distance (Siddon 1985). Projection data were generated and reconstructed using 128 views, 60 views, 21 views, 15 views and 9 views, uniformly distributed around 360 degrees. This system matrix was used to simulate data for the inverse crime case. This system matrix was also used in all reconstructions in this work.

A parametric sweep was performed to investigate the effects of the two parameters on the reconstructions: the TV weighting parameter, $\mathrm{Y}$, and the standard deviation of the Gaussian blurring kernel, $r$. Reconstructions were performed with $y$ varying from 0.0001 to 1.0 and $r$ varying from 0 to 2.0 pixels. For this case, $r_{\text {true }}$ is known to be equal to 0.75 . In practice, the amount of smoothness within the underlying object is unknown and may vary across the FOV. In this study, images are reconstructed using a range of $r$ values to quantify

Physics in Medicine and Biology, Vol. 58, No. 16 (August 2013): pg. 5629-5652. DOI. This article is (C) Institute of Physics and permission has been granted for this version to appear in e-Publications@Marquette. Institute of Physics does not grant permission for this article to be further copied/distributed or hosted elsewhere without the express permission from Institute of Physics. 
the performance of the reconstruction technique for the expected case where the assumed $r$ differs from $r_{\text {true }}$.

To reduce the necessary sampling for accurate image reconstruction, a sparse representation of an image must exist. Our proposed reconstruction approach assumes that the gradientmagnitude of the intermediate image $\boldsymbol{f}$ has very few meaningful coefficients. However, using an incorrect blurring model in the reconstruction may negatively affect the sparsity of the intermediate object, $\boldsymbol{f}$, limiting the benefits of the algorithm. To investigate the effect of the assumed blurring model on the sparsity of the reconstructed intermediate object, $\boldsymbol{f}$, images were reconstructed from 9 and 128 views using a range of $r$ values and sparsity evaluated as the number of pixels in the gradient-magnitude image of $\boldsymbol{f}$ with values greater than $10 \%$ of the maximum gradient magnitude of image $\boldsymbol{f}$.

To investigate the performance of the reconstruction technique in the presence of noise, simulations varying the number of views and parameter values were repeated with Poisson noise added to the projections generated from the system model. All simulations modeled approximately 1052000 counts, thus the peak number of counts in the 128 view projections was 298 while the peak number of counts in the 9 view projections was 3758. The noisy projection data were also reconstructed with MLEM in order to provide a reference reconstruction for comparison. As will be described in the next section, the correlation coefficient (CC) of the reconstructed image with the true object was used as a metric of accuracy throughout this work. In order to select a comparable stopping iteration for MLEM reconstruction, the CC was calculated at each MLEM iteration and the final image selected as that with the highest CC value.

\subsubsection{Metrics}

Evaluating the accuracy of the reconstructed object requires a measure of similarity or error between the reconstructed object and the true object. In SPECT imaging, including the Geant4 Application for Tomographic Emission (GATE) simulations proposed in section 4, the reconstructed activity is a scaled version of the true activity, with the scaling factor dependent on the geometric efficiency of the system (Jan et al 2004). Our reconstruction methods correct for the spatially

Physics in Medicine and Biology, Vol. 58, No. 16 (August 2013): pg. 5629-5652. DOI. This article is (C) Institute of Physics and permission has been granted for this version to appear in e-Publications@Marquette. Institute of Physics does not grant permission for this article to be further copied/distributed or hosted elsewhere without the express permission from Institute of Physics. 
varying sensitivity of the SPECT system, as will be described in section 4.1.2. However, a global scaling correction factor is not applied because absolute quantification in SPECT is challenging and may confound the characterization of the algorithm. Therefore, our accuracy metric must provide a meaningful measure of similarity in cases where the scaling factor between the reconstructed and true object is unknown. In this work, reconstruction accuracy was quantified using the correlation coefficient (CC) of the reconstructed image estimate with the true object. CC is defined as

$$
\mathrm{CC}=\frac{\sum_{k=1}^{M}(u(k)-\bar{u})\left(u_{\text {true }}(k)-\overline{u_{\text {true }}}\right)}{\left\{\sum_{k=1}^{M}(u(k)-\bar{u})^{2} \sum_{k=1}^{M}\left(u_{\text {true }}(k)-\overline{u_{\text {true }}}\right)^{2}\right\}^{1 / 2}},
$$

where $u_{\text {true }}$ is the true object, $M$ is the number of voxels and $u(k)$ is the reconstructed object value at voxel $k$. This metric is commonly used in image registration and is the optimum similarity measure for images that vary by a linear factor (Hill et al 2001). This metric allows the quantification of the accuracy of the spatial distribution of the object, without requiring absolute quantitative accuracy. CC is equal to one when the reconstructed object matches the true object. We also quantified the change in CC over the range of studied parameters ( $r$ and $\mathrm{Y}$ ), in order to quantify the sensitivity of the algorithm to parameter selections and to understand the performance of the algorithm when the assumed blurring parameter does not match the true object blur. Spatial resolution in the reconstructed images was quantified as the full-width at $10 \%$ of maximum (FW10M) of the central profile through the smaller disk. This measure was used instead of the more common full-width at half maximum (FWHM) because analysis of preliminary reconstructed images indicated that the FWHM was often accurate, even though the extent of the reconstructed object was greater than the true object. The FW10M more accurately quantified this blurring effect. The true object had a FW10M of 12 pixels. and permission has been granted for this version to appear in e-Publications@Marquette. Institute of Physics does not grant permission for this article to be further copied/distributed or hosted elsewhere without the express permission from Institute of Physics. 
NOT THE PUBLISHED VERSION; this is the author's final, peer-reviewed manuscript. The published version may be accessed by following the link in the citation at the bottom of the page.

\subsection{Results}

\subsubsection{Without Poisson noise}

We present results of the noiseless case in which the true object was constructed from the blurred piecewise constant object model and the projections were determined by multiplying the voxelized phantom (ground-truth object) by the system matrix. The purpose of this study was to confirm that the reconstruction algorithm closely approximated the true object in the noiseless inverse crime case and to examine the effects of the design parameters $r$ and $y$ as the number of views decreased. Both design parameters were varied and the number of angular samples reduced from 128 views to 9 views.

Figure 2 displays images reconstructed from nine views over the range of studied algorithm parameters. As seen in figure 2, blurring increased with increasing $r$ and/or $\gamma$. Artifacts were present in reconstructions with $r>1$ and $Y=0.0001$. Similar images resulted from 128 views (not shown), except that images reconstructed from 128 views exhibited fewer artifacts at $r>1$ and $\mathrm{y}=0.0001$.

Physics in Medicine and Biology, Vol. 58, No. 16 (August 2013): pg. 5629-5652. DOI. This article is @ Institute of Physics and permission has been granted for this version to appear in e-Publications@Marquette. Institute of Physics does not grant permission for this article to be further copied/distributed or hosted elsewhere without the express permission from Institute of Physics. 


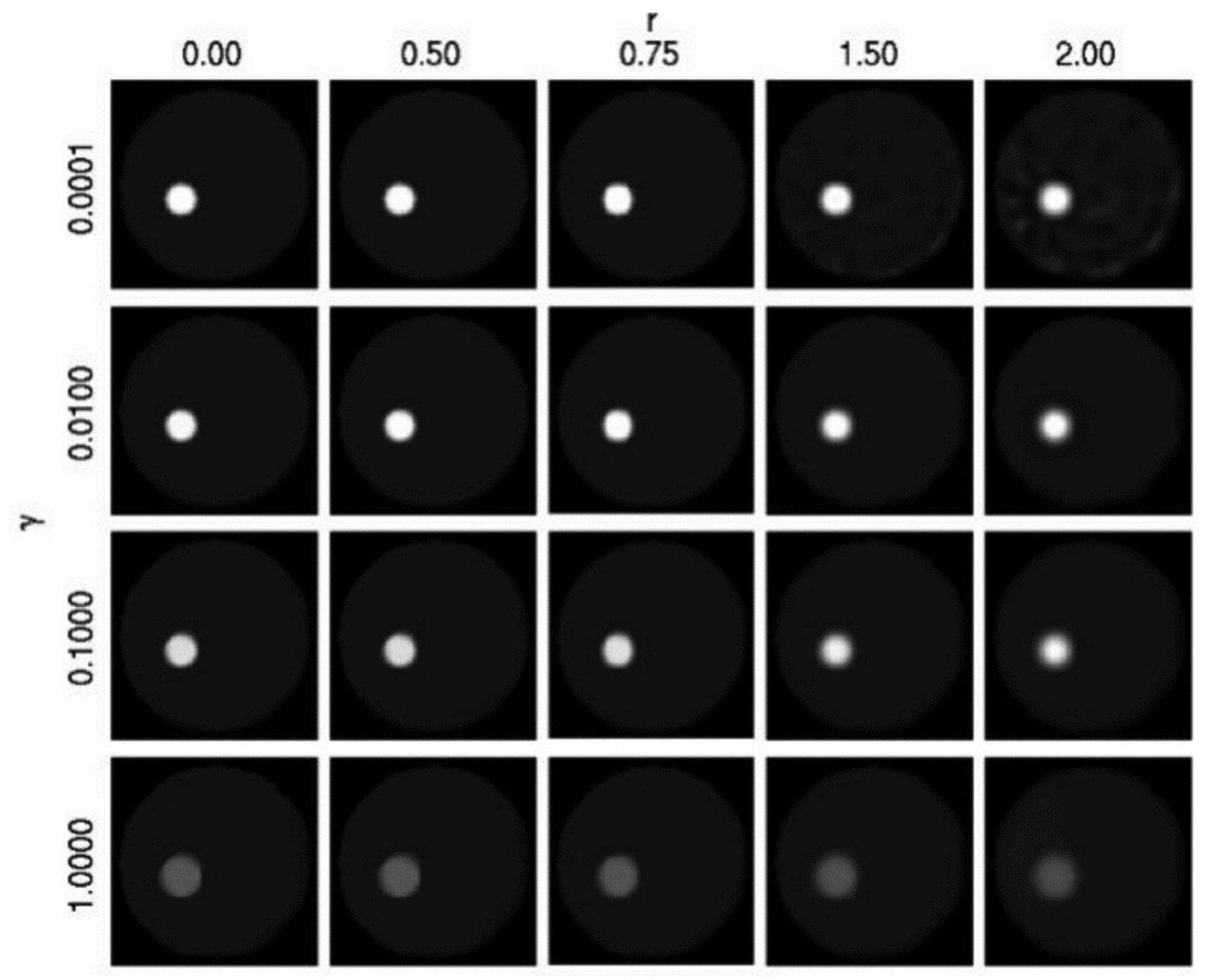

Figure 2.

Images reconstructed from 9 views of noiseless inverse crime data using the proposed algorithm with varying values of $r$ and $y$.

Figure 3 presents plots of CC over the range of studied $\mathrm{y}$ and $r$ values for images reconstructed from 128 views and nine views. For both the 128-view and nine view studies, reconstruction accuracy (CC) was high (CC $>0.980$ ) for reconstruction using $Y<1.0$, with $C C$ varying by less than $2 \%$ for all $r$ investigated. Using $Y=0.0001$ or $Y=$ 0.01 and $r=r_{\text {true }}=0.75$, the object was recovered nearly exactly with CC exceeding 0.999 in each case for the 128 view and nine view studies. Overall, in both the 128 and 9-view case, CC values demonstrated a larger range over the set of $\mathrm{Y}$ values compared to $r$ values, suggesting that the reconstruction technique is more sensitive to the selection of $\mathrm{y}$ than $r$. The FW10M value of the true object was 12 pixels, which was correctly depicted by reconstructions using $r<$ 1.0 and $Y<0.1$.

Physics in Medicine and Biology, Vol. 58, No. 16 (August 2013): pg. 5629-5652. DOI. This article is (C) Institute of Physics and permission has been granted for this version to appear in e-Publications@Marquette. Institute of Physics does not grant permission for this article to be further copied/distributed or hosted elsewhere without the express permission from Institute of Physics. 
a

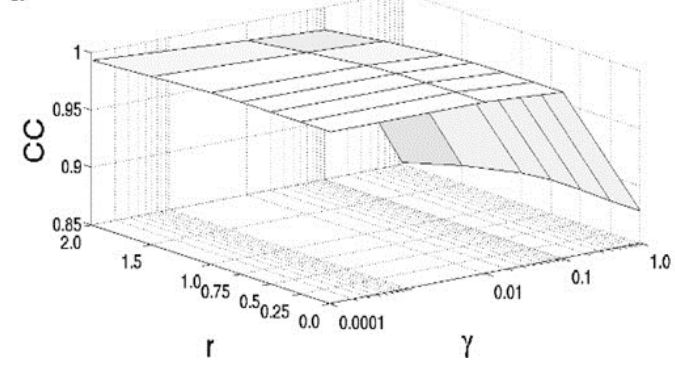

b

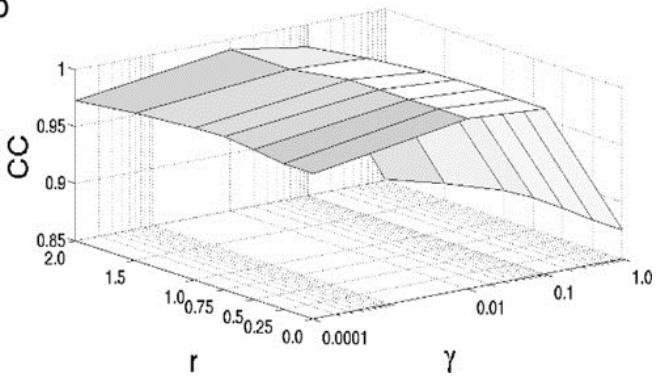

Figure 3.

Plots depicting the CC over the range of studied $r$ and $\mathrm{Y}$ parameters of images reconstructed from noiseless inverse crime data from 128 views (a) and 9 views (b).

Evaluating gradient magnitude sparsity of the intermediate image

This section evaluates the sparsity of the intermediate image $\boldsymbol{f}$ reconstructed from many-view and few-view data. Figure 4 shows images of the intermediate image, $\boldsymbol{f}$, reconstructed from both 128 views and 9 views using different $r$ and $Y=0.0001$. Each image is captioned by its sparsity value (number of meaningful coefficients).

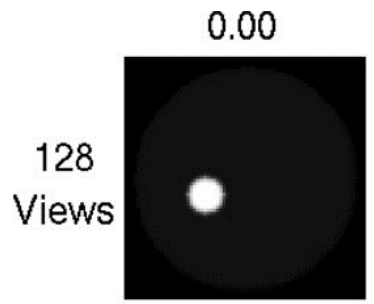

388

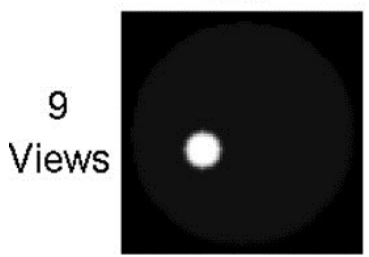

393
0.50

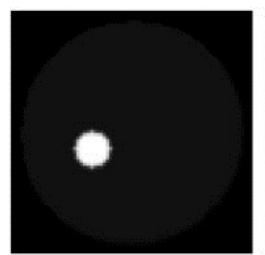

370

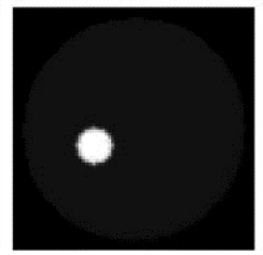

354
0.75

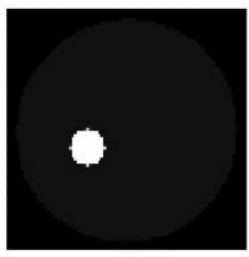

243

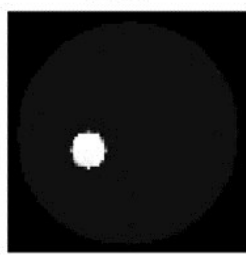

240
1.50

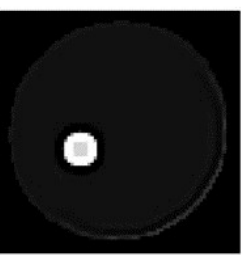

288

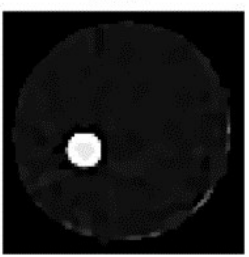

262

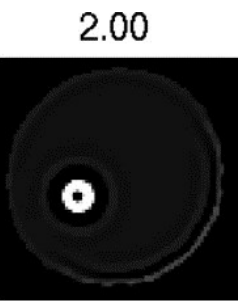

317

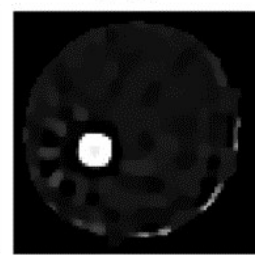

508

Figure 4.

Intermediate images $\boldsymbol{f}$ and the number of meaningful sparsity coefficients reconstructed from 128 and 9 noiseless inverse crime data using $\mathrm{Y}=0.0001$.

In both the many-view and few-view case, the image reconstructed with the true blurring model $(r=0.75)$ was the most sparse and as the $r$ assumed by the algorithm diverged from $r_{\text {true }}$ the images became less sparse. This indicates that using the correct blurring model may allow the greatest sampling reductions.

Physics in Medicine and Biology, Vol. 58, No. 16 (August 2013): pg. 5629-5652. DOI. This article is @ Institute of Physics and permission has been granted for this version to appear in e-Publications@Marquette. Institute of Physics does not grant permission for this article to be further copied/distributed or hosted elsewhere without the express permission from Institute of Physics. 
Additionally, underestimating $r$ leads to a gradual increase in the number of meaningful coefficients. In the few-view case, overestimating $r$ leads to a rapid increase in the number of meaningful coefficients, reflected by the fact that new structure enters the image. These artifacts survive the blurring with $\boldsymbol{G}$, leading to artifacts in the presented image $\boldsymbol{u}$.

\subsubsection{With Poisson noise added}

We next considered data generated by the system matrix with the addition of Poisson noise. The purpose of this study was to examine the effects of noise on the reconstructions, using data from an otherwise inverse crime case, in which the object model and system model are known exactly. Figure 5 shows images reconstructed from nine views over the range of $r$ and $y$ parameters. The images reconstructed from 128 views (not shown) demonstrated similar trends, with the exception that the noise structure at $\mathrm{y}=$ 0.0001 demonstrated more noise and higher frequency noise content at 128 views. One possible explanation for the higher noise at 128 views is that nine-views was sufficient for reconstructing this simple object, while the nine-vew case contained less noise in each projection compared to the 128 view case. Section 4.2.2 presents results from a different phantom where the number of counts per view was held constant as the view sampling decreased. Figure 5 shows that the manifestation of noise in the reconstructed images is affected by both $\mathrm{Y}$ and $r$. Images reconstructed with low $\mathrm{Y}$ values $(\mathrm{Y}=0.0001)$ demonstrated streaking artifacts at lower $r$ values. As in the noiseless case, contrast and spatial resolution decreased with increasing $\mathrm{Y}$. 


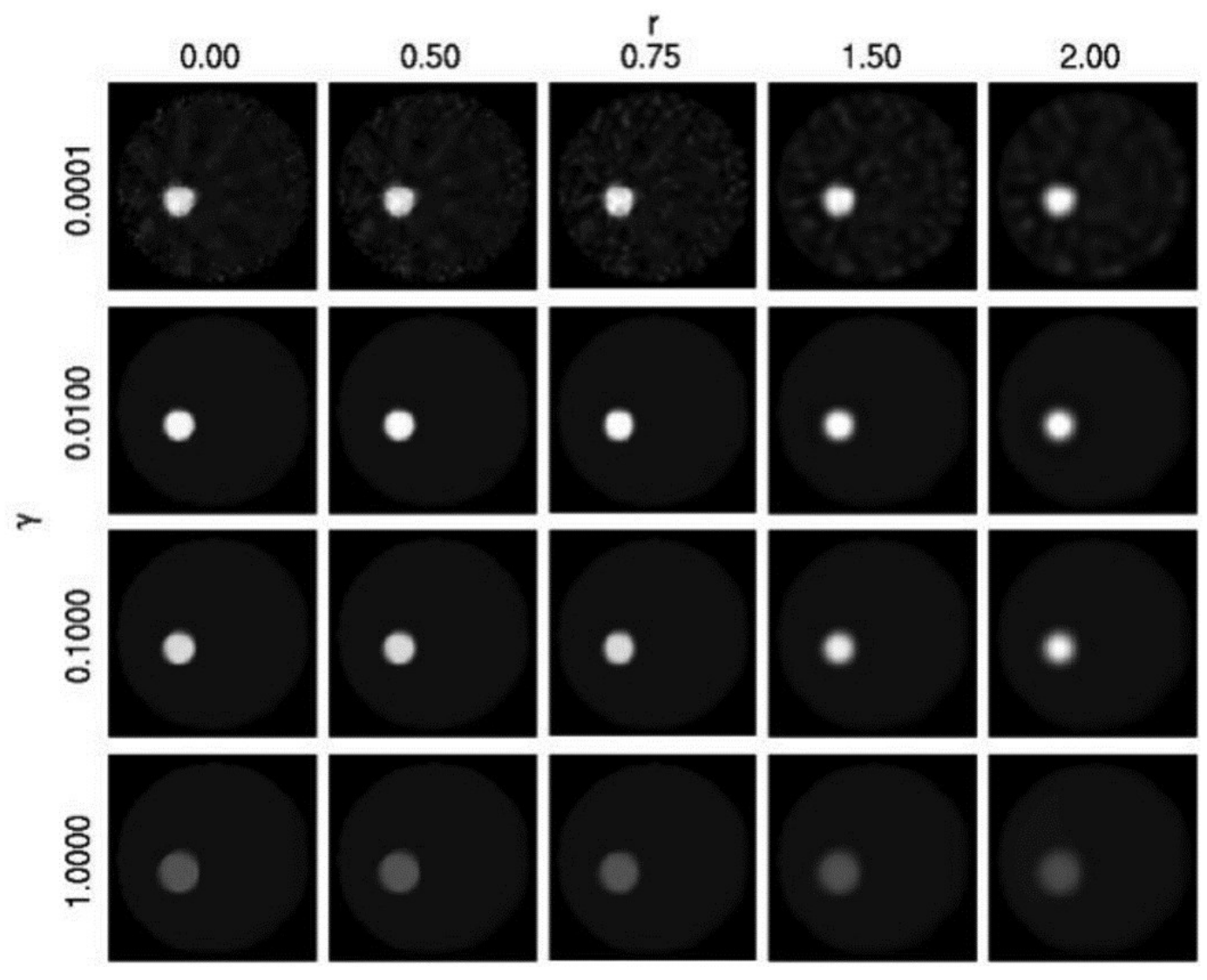

\section{Figure 5.}

Images reconstructed from 9 views of noisy data using the proposed algorithm with varying values of $r$ and $\mathrm{Y}$. For these images, the projection data were generated by the system matrix.

Figure 6 plots the CC metric over the range of studied parameters for noisy images reconstructed from 128 and nine views. As in the noiseless case, the CC varied by less than $1.5 \%$ across the studied $r$ values for $\mathrm{Y}>0.0001$ and 128 views. Unlike the noiseless case, when $Y=0.0001$, the CC increased from 0.867 to 0.988 as $r$ increased from 0.0 to 2.0, as the increased blurring provided additional regularity and noise reduction. Noise is also reduced as $Y$ is increased, due to the increased weighting of the TV term. The highest CC value of 0.999 for the 128 -view case and 0.997 for the nine-view case occurred when $r=0.75$, the true value of $r$, and $\gamma=0.01$. Overall, as in the noiseless case, CC demonstrated greater variation with $\mathrm{Y}$ compared to $r$, and both the 9- and 128-view reconstructions suggest that $\mathrm{Y}=0.01$ provides the highest $\mathrm{CC}$. and permission has been granted for this version to appear in e-Publications@Marquette. Institute of Physics does not grant permission for this article to be further copied/distributed or hosted elsewhere without the express permission from Institute of Physics. 
a

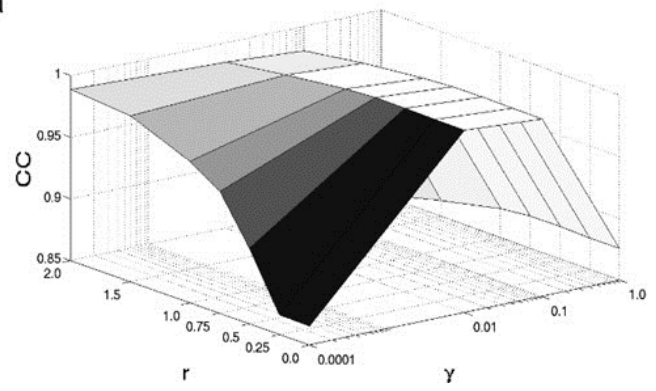

b

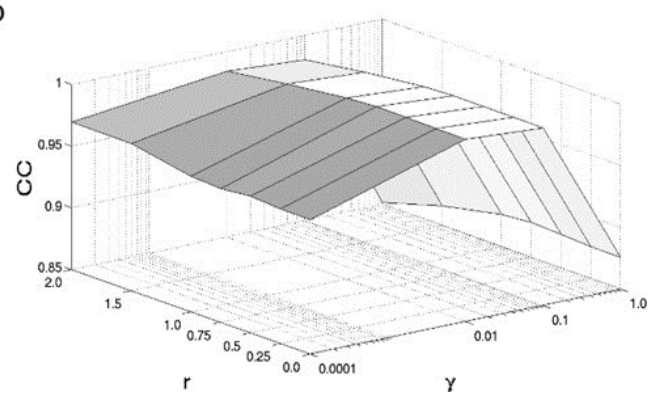

Figure 6.

Plots depicting the CC over the range of studied $r$ and $\mathrm{y}$ parameters of images reconstructed from noisy data from 128 views (a) and 9 views (b). For these images, the projection data were generated by the system matrix.

Figure 7 compares images reconstructed with the proposed reconstruction technique and MLEM from noisy data acquired with a varying number of angular views. The displayed images in figure 7 were reconstructed with $y=0.01$ and $r=0.75$. The true and in practice unknown value for $r$ was used so the best case can be examined. Table 1 lists the CC and FW10M values for each reconstruction technique and number of views. Images reconstructed using the proposed algorithm had CC values that were $2-4 \%$ higher for each case compared to MLEM. However, these reconstructions used knowledge of the true blurring parameter. Sections 4 and 5 investigate reconstructions where the blurring parameter is undetermined. Streak artifacts were present in the MLEM reconstructions, and were primarily absent in images reconstructed with the proposed algorithm. Both algorithms provide similar FW10M values compared to the true value of 12 .

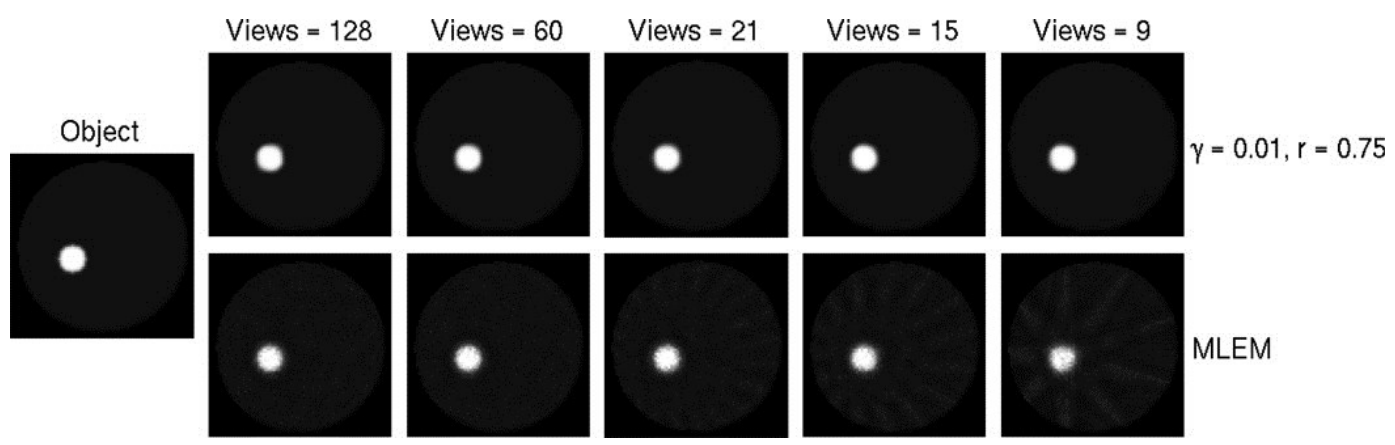

Figure 7.

Images reconstructed from noisy projections using the proposed algorithm and MLEM for varying sampling cases. For these images, the projection data were generated by the system matrix.

Physics in Medicine and Biology, Vol. 58, No. 16 (August 2013): pg. 5629-5652. DOl. This article is (C) Institute of Physics and permission has been granted for this version to appear in e-Publications@Marquette. Institute of Physics does not grant permission for this article to be further copied/distributed or hosted elsewhere without the express permission from Institute of Physics. 
NOT THE PUBLISHED VERSION; this is the author's final, peer-reviewed manuscript. The published version may be accessed by following the link in the citation at the bottom of the page.

\begin{tabular}{lllllll}
\hline & & 128 views & 60 views & 21 views & 15 views & 9 views \\
\hline$\gamma=0.010$ & CC & 0.999 & 0.998 & 0.998 & 0.998 & 0.999 \\
$r=0.75$ & FW10M & 12 & 12 & 12 & 12 & 12 \\
\hline MLEM & CC & 0.986 & 0.987 & 0.984 & 0.981 & 0.973 \\
& FW10M & 13 & 13 & 14 & 12 & 15 \\
\hline
\end{tabular}

Table 1.

Comparison of image quality metrics from images reconstructed from noisy projections generated by the system matrix.

\section{Monte Carlo simulation study with contrast element phantom}

The purpose of this study was to characterize the performance of the reconstruction technique for the more realistic case where the object does not necessarily match the model assumed in reconstruction, and the modeled system matrix is an approximation to the system that generated the data. In addition, these simulations include realistic effects such as scatter and spatially-varying pinhole sensitivity and blurring.

\subsection{Methods}

\subsubsection{Phantom}

The object was defined on a $512 \times 512$ pixel grid of $0.25 \times 0.25$ $\mathrm{mm}$ pixels. The object consisted of a $28 \mathrm{~mm}$-radius disk of background activity containing five contrast elements of varying size, shape, and intensity, as detailed in Table 2 and displayed in figure 8. Two, twodimensional Gaussian distributions with peak intensities $638 \mathrm{~Bq}$ and $319 \mathrm{~Bq}$ and standard deviations $4 \mathrm{~mm}$ and $8 \mathrm{~mm}$ truncated to have radius $4.4 \mathrm{~mm}$ were embedded in the larger disk. Also included in the phantom was a disk representing a cold region with radius $4.4 \mathrm{~mm}$ and one disk with radius $2.2 \mathrm{~mm}$ having constant intensity, as detailed in Table 2 . The activity levels were selected to approximately match the range of activity measured in a previous experimental rat lung study (Ma et al 2013). None of the elements in the phantom were generated by the smoothed piecewise constant model assumed by the

Physics in Medicine and Biology, Vol. 58, No. 16 (August 2013): pg. 5629-5652. DOI. This article is (C) Institute of Physics and permission has been granted for this version to appear in e-Publications@Marquette. Institute of Physics does not grant permission for this article to be further copied/distributed or hosted elsewhere without the express permission from Institute of Physics. 
NOT THE PUBLISHED VERSION; this is the author's final, peer-reviewed manuscript. The published version may be accessed by following the link in the citation at the bottom of the page.

reconstruction algorithm, thus representing a challenging reconstruction task.

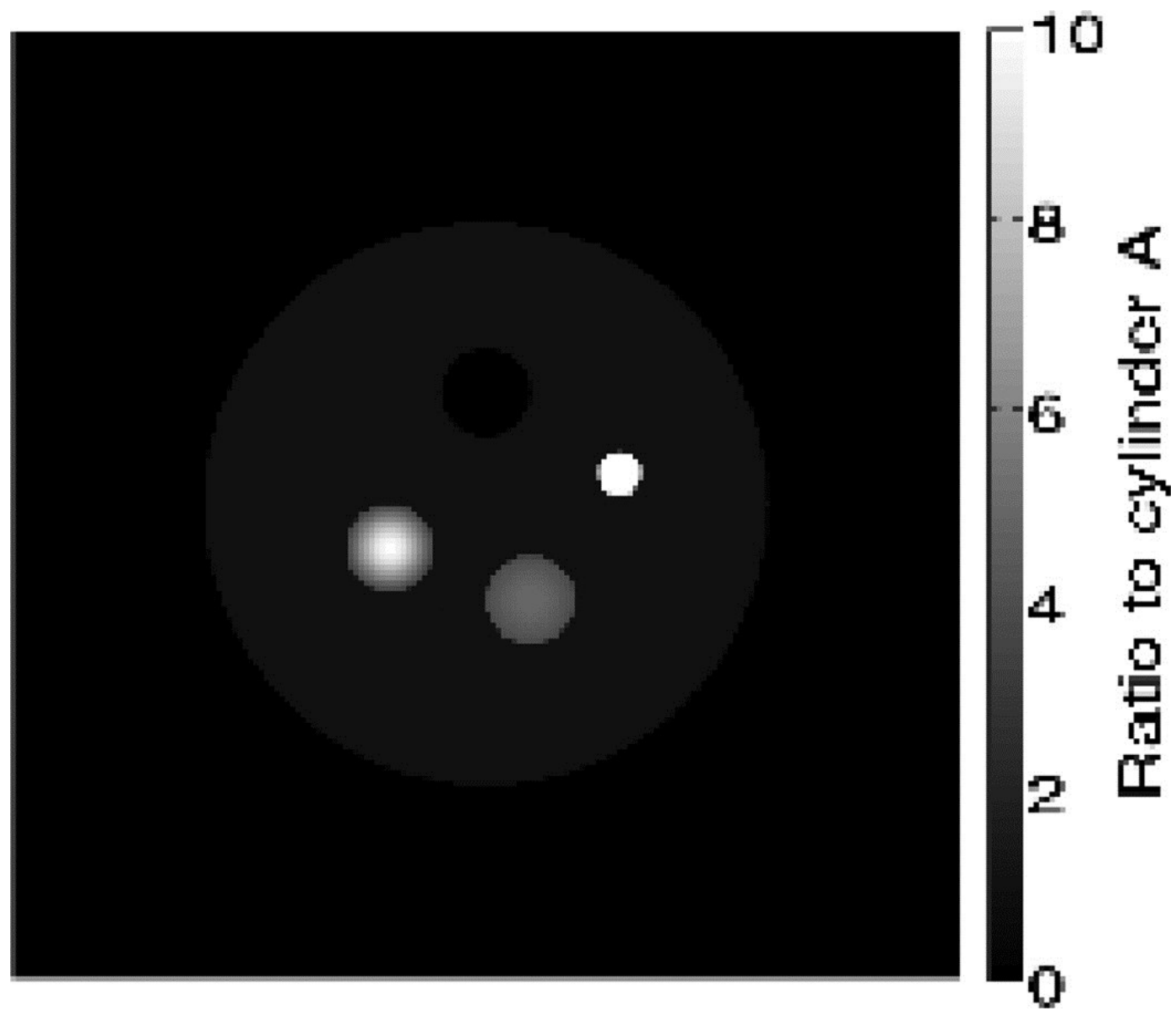

\section{Figure 8.}

Voxelized phantom used in the GATE studies. The phantom contains contrast elements of varying shape and size as described in Table 2.

\begin{tabular}{llll}
\hline Element & Radius $(\mathrm{mm})$ & Position $(\mathrm{mm})$ & Intensity (relative) \\
\hline $\mathrm{A}$ & 28 & $(0,0)$ & Constant; $64 \mathrm{~Bq} /$ pixel \\
$\mathrm{B}$ & 4.4 & $(-13,6)$ & Peak $=638 \mathrm{~Bq} /$ pixel; Std Dev $=4 \mathrm{~mm}$ \\
$\mathrm{C}$ & 4.4 & $(6,-13)$ & Peak $=319 \mathrm{~Bq} /$ pixel; Std Dev $=8 \mathrm{~mm}$ \\
$\mathrm{D}$ & 4.4 & $(0,15)$ & 0 \\
$\mathrm{E}$ & 2.2 & $(18,4)$ & Constant; $640 \mathrm{~Bq} /$ pixel \\
\hline
\end{tabular}

Table 2.

GATE Phantom Specifications.

Physics in Medicine and Biology, Vol. 58, No. 16 (August 2013): pg. 5629-5652. DOI. This article is @ Institute of Physics and permission has been granted for this version to appear in e-Publications@Marquette. Institute of Physics does not grant permission for this article to be further copied/distributed or hosted elsewhere without the express permission from Institute of Physics. 
NOT THE PUBLISHED VERSION; this is the author's final, peer-reviewed manuscript. The published version may be accessed by following the link in the citation at the bottom of the page.

\subsubsection{Simulations}

Projections of the pixelized object were generated using GATE Monte Carlo simulation to model the stochastic emission of photons from a voxelized phantom and their stochastic transmission through the collimator and camera. A three-camera system was simulated. Each collimator was simulated as a $20 \mathrm{~mm}$ thick tungsten plate having a $3 \mathrm{~mm}$ diameter pinhole with $1.5 \mathrm{~mm}$ channel length. A $128 \mathrm{~mm} \times 1$ $\mathrm{mm} \mathrm{NaI}$ crystal was simulated and detected photons binned into $1 \mathrm{~mm}$ $x 1 \mathrm{~mm}$ pixels. Compton scatter, Rayleigh scatter and photoelectric absorption were included as possible interactions for $140 \mathrm{keV}$ photons. Photons detected outside the 129.5 - $150.5 \mathrm{keV}$ range were rejected as scatter. To reduce computational load and to enable investigation of multiple parameter settings, only 2D simulations were performed for this preliminary study of algorithm feasibility. Thus, the object was modeled in GATE as one slice of a voxelized phantom, and the detector was modeled as a single row. Electronic noise was not modeled. The system is described in figure 9 and table 3. 


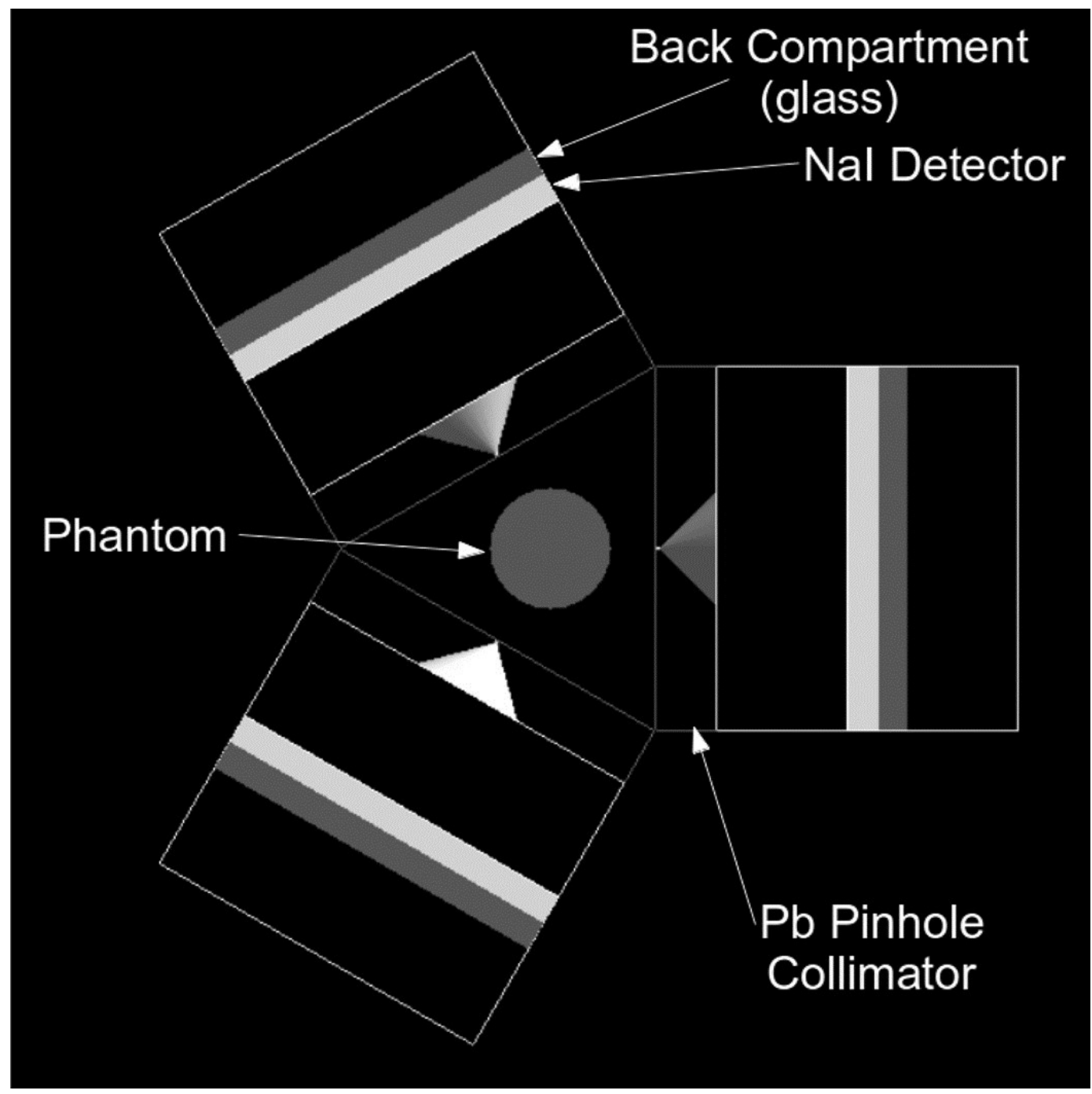

\section{Figure 9.}

Diagram of Simulated SPECT system. The contrast-element phantom used in this study fills the FOV seen by all three pinholes.

\begin{tabular}{lc}
\hline Camera size & $128 \mathrm{~mm} \times 120 \mathrm{~mm} \times 1 \mathrm{~mm}$ \\
Pinhole diameter & $3 \mathrm{~mm}$ \\
Pinhole-to-object center distance & $35 \mathrm{~mm}$ \\
Pinhole-to-detector distance & $63.5 \mathrm{~mm}$ \\
\hline
\end{tabular}

Table 3.

Specifications of the simulated SPECT system.

The sensitivity of pinhole collimators depends on the angle of the ray incident on the pinhole. In order to correct for the spatially-

Physics in Medicine and Biology, Vol. 58, No. 16 (August 2013): pg. 5629-5652. DOI. This article is (C) Institute of Physics and permission has been granted for this version to appear in e-Publications@Marquette. Institute of Physics does not grant permission for this article to be further copied/distributed or hosted elsewhere without the express permission from Institute of Physics. 
varying pinhole sensitivity during reconstruction, a sensitivity map was generated by simulating a flood source on the collimator surface (Vanhove et al 2008). The resulting projection represents the spatiallyvarying sensitivity of the pinhole and was incorporated into the reconstruction algorithm. The sensitivity map was multiplied during each forward projection prior to the summing of data from each ray. Data were multiplied by the sensitivity map prior to backprojection. The system matrix used in reconstruction was calculated as described in Section 3.

Two distinct cases were simulated. In the first case, the total simulated scan time was held constant as the number of views decreased in order to examine the effects of angular undersampling independent of changes in noise. Scans comprising 60,21, 15, and 9 views distributed over 360 degrees acquired during a 200 second scan were simulated. These data had approximately the same number of total counts in each simulation ( $~ 65000$ counts). The noise level in SPECT imaging is dependent on the number of detected counts, so the reconstructed images should have similar noise statistics regardless of the number of view angles. The second simulated case held the acquisition time of each view constant across all angular sampling cases. By doing so, the scans that used fewer views had improved temporal sampling, but fewer counts. As the number of views decreased, so did the absolute intensities of the reconstructed images. Images were acquired over 10 seconds for each position of the threecamera gantry, thereby varying the total scan time from 200 seconds for 60 views to 30 seconds for 9 views. In this case, the simulated scan with the fewest views (9) had the fewest counts ( 10000 counts) and, consequently, the highest noise level. This represents a more realistic approach for providing dynamic scans with high temporal sampling.

The simulated phantom cannot be described using a constant $r$ across the spatial domain. Each disk has a definite edge and distinct profile. To investigate the effects of varying $r$ in the case where its optimal value is unknown, data were reconstructed using the TV case $(r=0.0)$ and varying $r$ from 0.25 to 2.0 pixels. The TV weighting parameter was varied from 0.0001 to 1.0 . Images were reconstructed onto pixels of size $0.5 \times 0.5 \mathrm{~mm}$. The resulting images were evaluated on the basis of reconstruction accuracy with the CC metric as and permission has been granted for this version to appear in e-Publications@Marquette. Institute of Physics does not grant permission for this article to be further copied/distributed or hosted elsewhere without the express permission from Institute of Physics. 
NOT THE PUBLISHED VERSION; this is the author's final, peer-reviewed manuscript. The published version may be accessed by following the link in the citation at the bottom of the page.

described in section 3.1.3. The phantom was resampled to a grid of reconstruction pixels $(0.5 \mathrm{~mm} \times 0.5 \mathrm{~mm})$ and used as the "true" image in the CC calculation Spatial resolution was quantified by considering FW10M of a profile through the center of disk $\mathrm{E}$, as the piecewise constant shape provided a step edge at which to evaluate spatial resolution. In each case, MLEM reconstructions are also presented as a reference, with the MLEM stopping iteration selected as the iteration with the highest CC value.

\subsection{Results}

In this section we present the results of the Monte Carlo simulations performed over a range of angular sampling schemes for two different cases: constant total scan time and constant scan time per view.

\subsubsection{Constant total scan time}

Data reconstructed using the proposed algorithm from 60 views and a range of $r$ and $\mathrm{Y}$ values are presented in figure 10 with plots of $\mathrm{CC}$ in figure 11. 


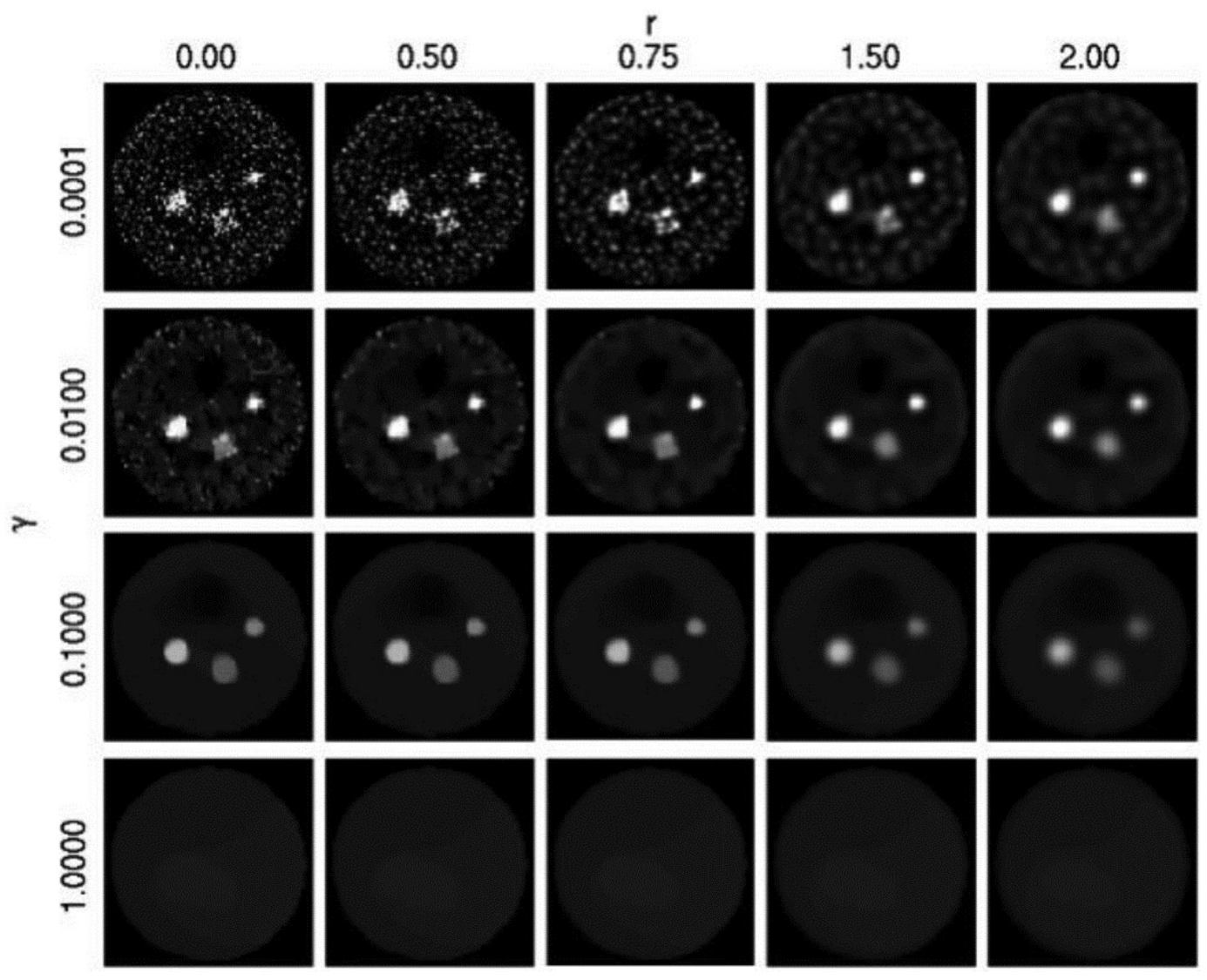

Figure 10.

Images reconstructed from 60 views of GATE data simulated for 200 seconds using the proposed algorithm with varying values of $r$ and $Y$.

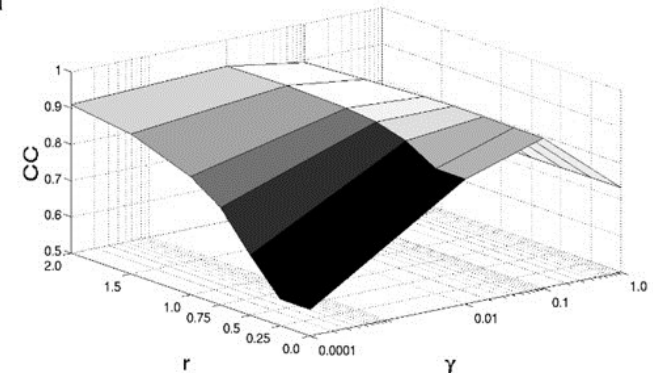

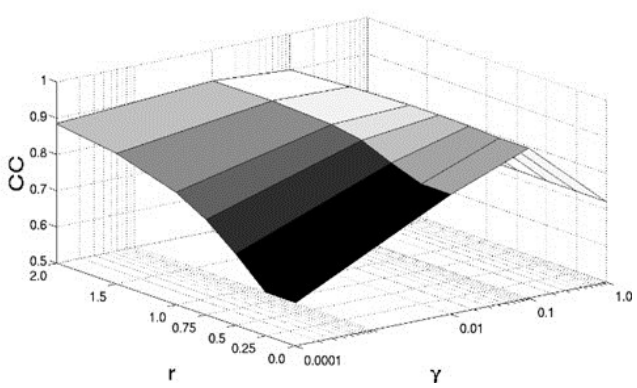

Figure 11.

Plots depicting the CC over the range of studied $r$ and $\mathrm{Y}$ parameters of images reconstructed from GATE data simulated for 200 seconds, using 60 views (a) and 9 views (b).

When the TV weighting parameter was small $(\gamma=0.0001)$, the resulting image contained high frequency noise; when the TV weighting parameter was large $(\gamma=1.0)$, the object was blurred and

Physics in Medicine and Biology, Vol. 58, No. 16 (August 2013): pg. 5629-5652. DOI. This article is (C) Institute of Physics and permission has been granted for this version to appear in e-Publications@Marquette. Institute of Physics does not grant permission for this article to be further copied/distributed or hosted elsewhere without the express permission from Institute of Physics. 
contrast reduced. The remainder of the results will focus on $Y=0.1$ and $Y=0.01$. When $Y=0.1$, the reconstructed objects did not depict the true contrast of the phantom for any values of $r$. Using $Y=0.01$, the contrast is more accurately depicted but the CC of the reconstructed images is more sensitive to the selection of $r$. The MLEM reconstructions also did not depict the true values of constrast, suggesting that this error may be caused by system blurring rather than the reconstruction algorithm. Using $Y=0.01$, the $r$ value that yielded the optimal image (highest CC) was 1.5, compared to an optimal $r$ value of 0.75 when $\mathrm{y}=0.1$.

Figure 12 displays the images reconstructed from 9 views, which demonstrated behavior similar to images reconstructed using 60 views. Images reconstructed using $Y=0.01$ contained more noise than images reconstructed using $Y=0.1$.
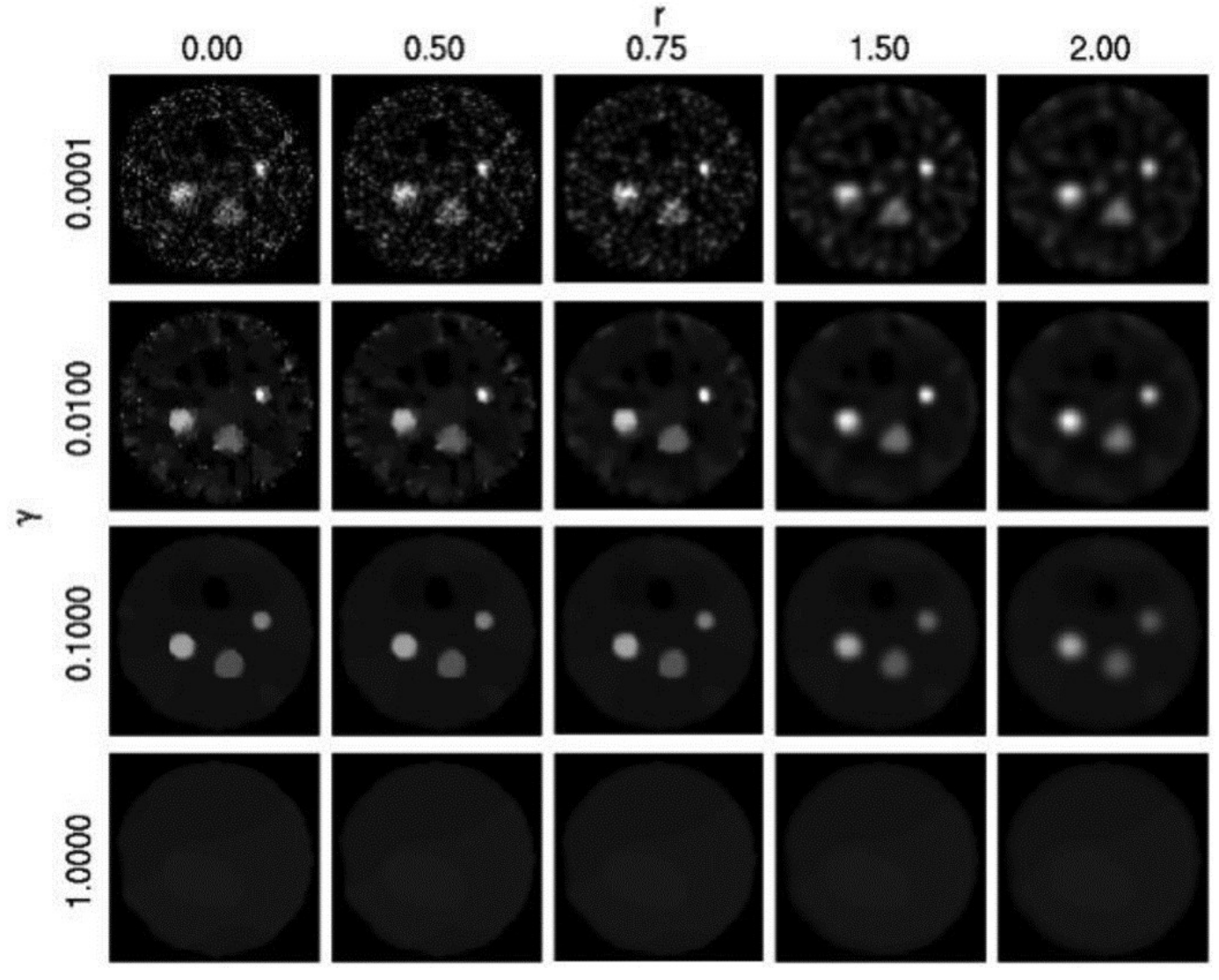

Figure 12.

Images reconstructed from 9 views of GATE data simulated for 200 seconds using the proposed algorithm with varying values of $r$ and $y$.

Physics in Medicine and Biology, Vol. 58, No. 16 (August 2013): pg. 5629-5652. DOI. This article is (C) Institute of Physics and permission has been granted for this version to appear in e-Publications@Marquette. Institute of Physics does not grant permission for this article to be further copied/distributed or hosted elsewhere without the express permission from Institute of Physics. 
Figure 13 compares images reconstructed from a varying number of views using the proposed algorithm with parameter pairs that provided the highest $\mathrm{CC}(\mathrm{y}=0.01, r=1.5$ and $\mathrm{y}=0.1, r=0.75)$ and MLEM. For images reconstructed using the proposed reconstruction technique with $\mathrm{Y}=0.01$ and $r=1.50$, the CC of the images varied by less than $1 \%$ from 0.946 to 0.942 as the number of views decreases from 60 to 9 . The CC varied similarly for images reconstructed using $\mathrm{Y}=0.1$ and $r=0.75$. For comparison, the CC of images reconstructed by MLEM decreased $6.5 \%$ from 0.913 to 0.854 as the number of views decrease from 60 to 9 . For this object, the proposed reconstruction algorithm using both $\mathrm{Y}=0.01$ and $\mathrm{Y}=0.1$ provided higher CC compared to MLEM for all angular cases, while providing similar FW10M values. Noise is manifest as low-frequency patchy artifacts in the background of images reconstructed using the proposed method. Streak artifacts, present in the MLEM reconstructions are reduced using the proposed reconstruction algorithm.

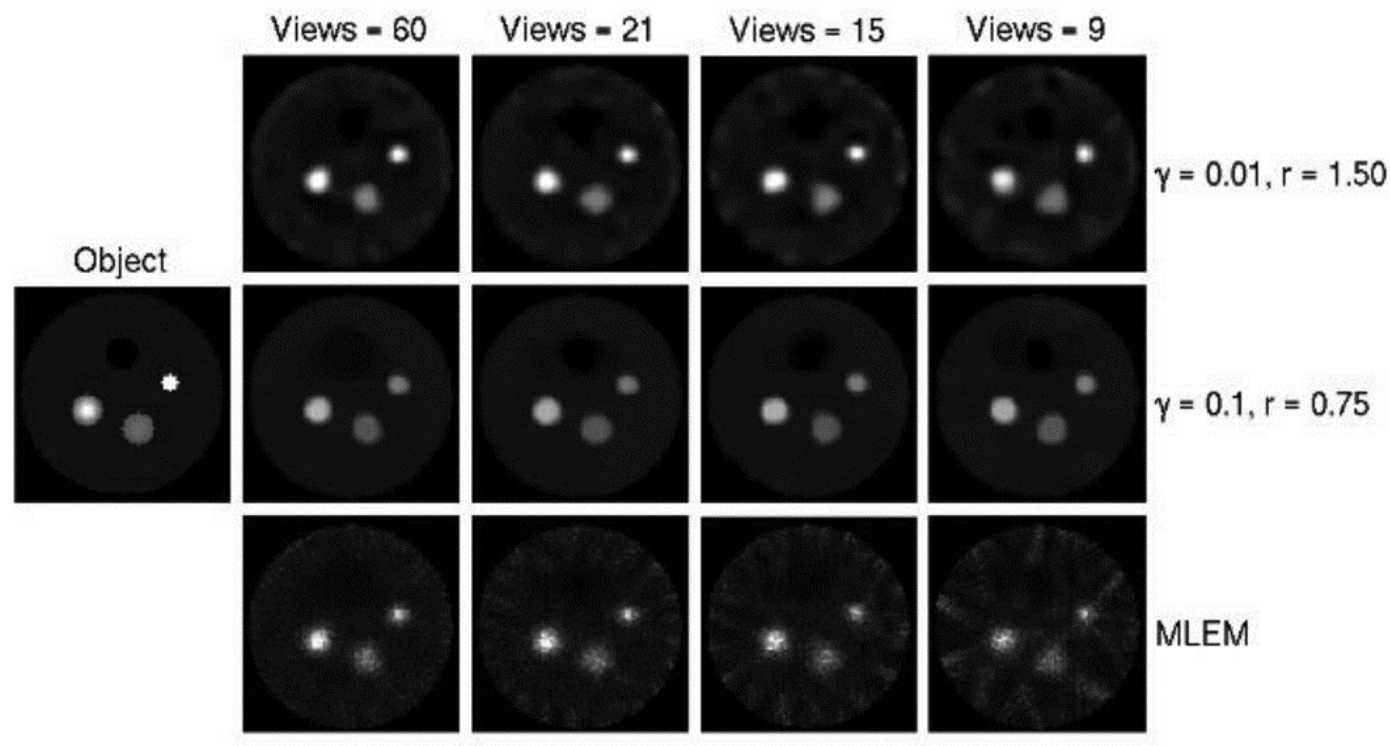

Figure 13.

Reconstructions of GATE data simulated for 200s over different numbers of angles using the proposed algorithm and MLEM.

\subsubsection{Constant scan time per view}

This set of simulations modeled a constant scan time per view (i.e., decreasing total scan time with decreasing number of views),

Physics in Medicine and Biology, Vol. 58, No. 16 (August 2013): pg. 5629-5652. DOI. This article is (C) Institute of Physics and permission has been granted for this version to appear in e-Publications@Marquette. Institute of Physics does not grant permission for this article to be further copied/distributed or hosted elsewhere without the express permission from Institute of Physics. 
NOT THE PUBLISHED VERSION; this is the author's final, peer-reviewed manuscript. The published version may be accessed by following the link in the citation at the bottom of the page.

representing the case where temporal sampling improves as the number of views decreases. Figure 14 presents images reconstructed from 9 views with 10 seconds per view (compared to 66.67 seconds per view in figures $11 \mathrm{~b}, 1212$ and and13),13), while figure 15 plots the $\mathrm{CC}$ across the range of $\mathrm{r}$ and gamma.
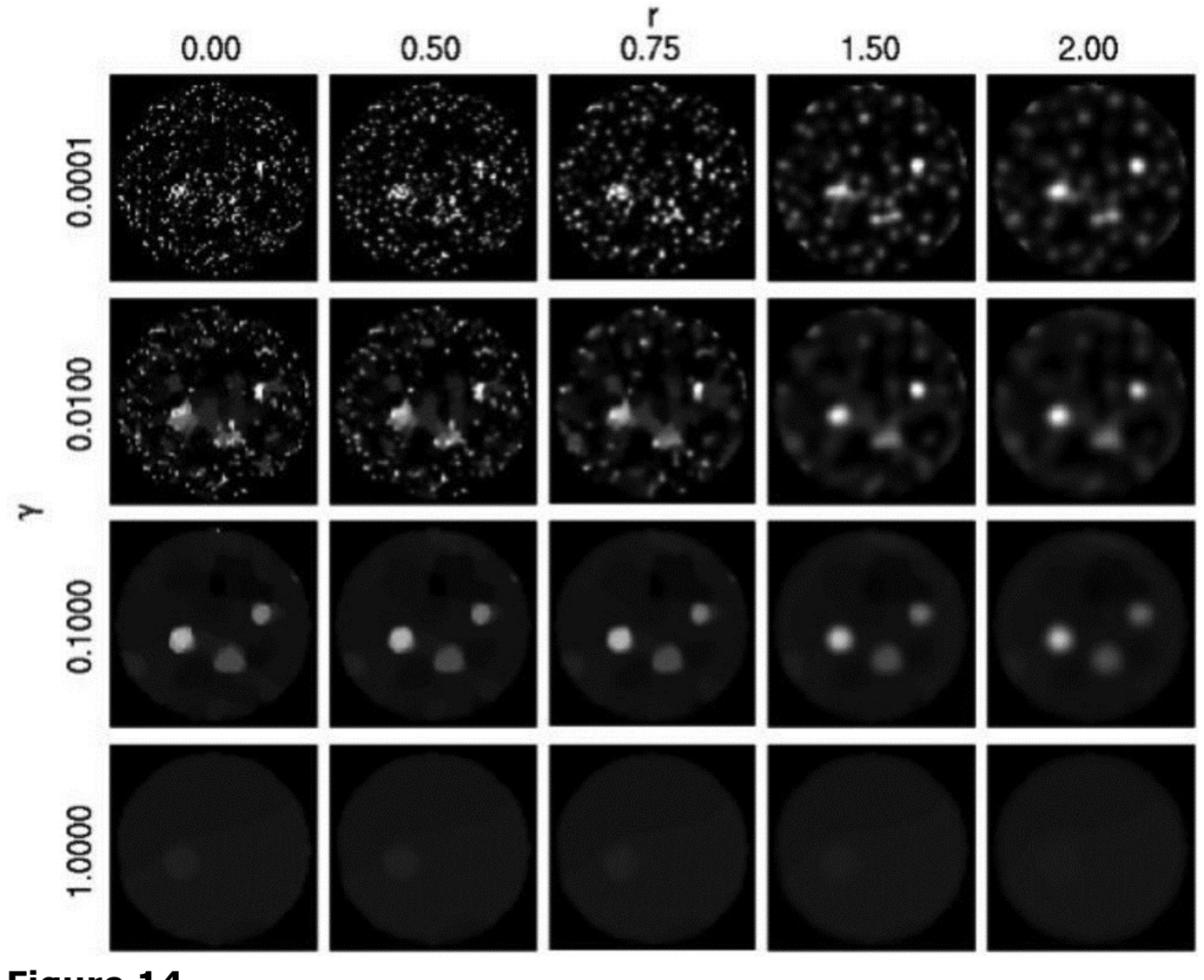

Figure 14.

Images reconstructed from 9 views of GATE data simulated for 30 seconds using the proposed algorithm with varying values of $r$ and $Y$.

Physics in Medicine and Biology, Vol. 58, No. 16 (August 2013): pg. 5629-5652. DOI. This article is @ Institute of Physics and permission has been granted for this version to appear in e-Publications@Marquette. Institute of Physics does not grant permission for this article to be further copied/distributed or hosted elsewhere without the express permission from Institute of Physics. 


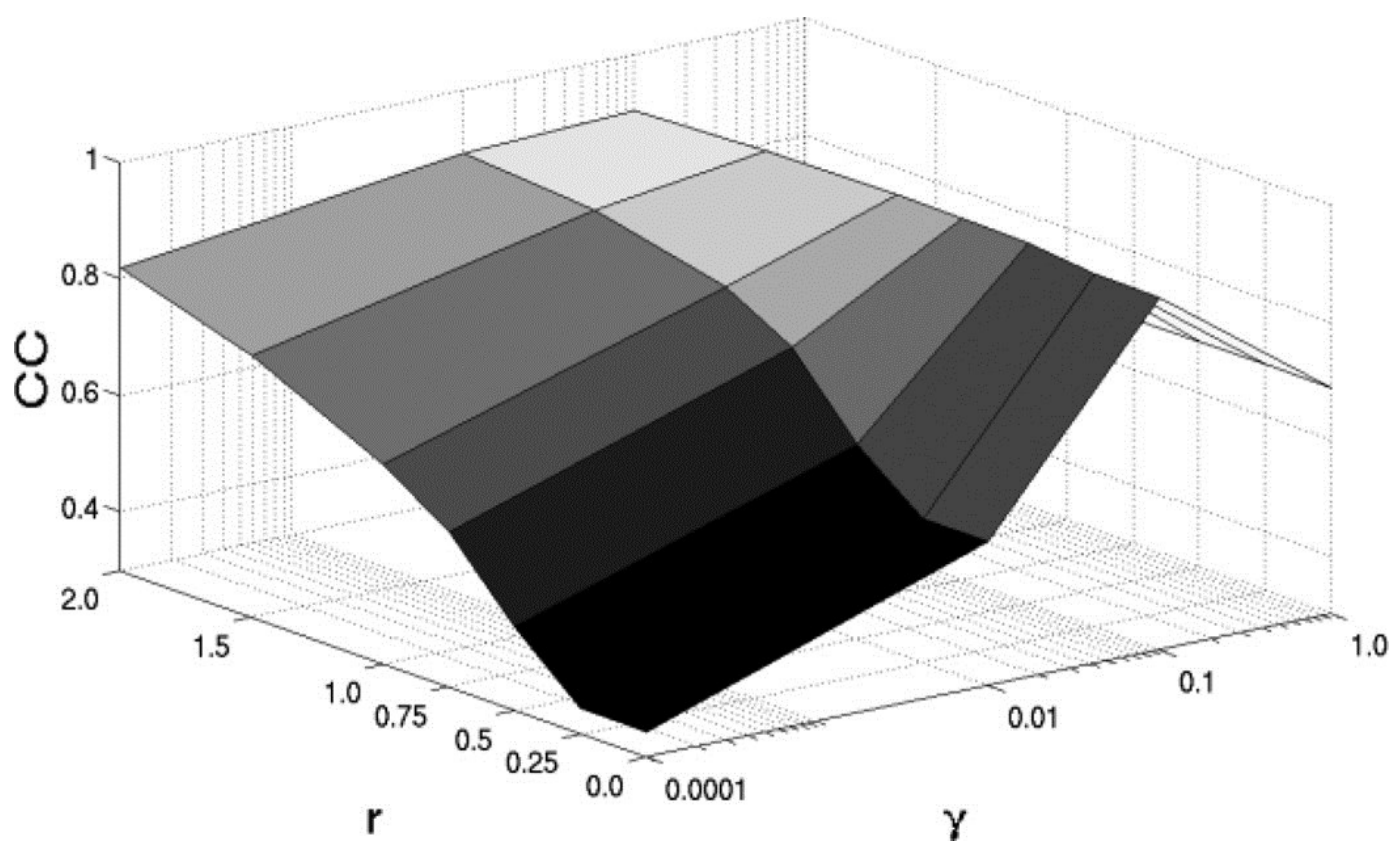

Figure 15.

Plot depicting the CC over the range of studied $r$ and $\mathrm{y}$ parameters of images reconstructed from GATE data simulated for 9 views over 30 seconds.

When comparing the $\mathrm{CC}$ values plotted in figure 15 with the $\mathrm{CC}$ values in figure $11 \mathrm{~b}$, it is apparent that images reconstructed from nine views and 10 seconds per view using $Y=0.01$ had lower reconstruction accuracy $(\mathrm{CC}<0.9)$ compared to the images reconstructed from a nine views and 66.67 seconds per view. This result of lower CC in the case of constant scan time per view is also evident when comparing the $\mathrm{CC}$ values listed in table 4 with the values in table 5. Using $\mathrm{Y}=0.1$, a maximum CC value of 0.921 occurred when $r=0.75$. Unlike the 66.67 seconds per view scans, the 10 seconds per view scans showed a larger variation in CC ( $40 \%)$ across the range of $r$ values when $y$ was not equal to 0.1 .

Physics in Medicine and Biology, Vol. 58, No. 16 (August 2013): pg. 5629-5652. DOI. This article is @ Institute of Physics and permission has been granted for this version to appear in e-Publications@Marquette. Institute of Physics does not grant permission for this article to be further copied/distributed or hosted elsewhere without the express permission from Institute of Physics. 


\begin{tabular}{llllll}
\hline & & 60 views & 21 views & 15 views & 9 views \\
\hline$\gamma=0.01, r=1.50$ & CC & 0.946 & 0.945 & 0.942 & 0.946 \\
& FW10M & 7 & 8 & 9 & 8 \\
$\gamma=0.10, r=0.75$ & CC & 0.942 & 0.940 & 0.941 & 0.945 \\
& FW10M & 8 & 8 & 9 & 9 \\
MLEM & CC & 0.913 & 0.901 & 0.889 & 0.854 \\
& FW10M & 8 & 11 & 9 & 8 \\
\hline
\end{tabular}

\section{Table 4.}

Comparison of image quality metrics for images reconstructed from GATE data with the total scan time held constant as the number views decreased.

\begin{tabular}{llllll}
\hline & & 60 views & 21 views & 15 views & 9 views \\
\hline$\gamma=0.01, r=1.50$ & CC & 0.946 & 0.915 & 0.889 & 0.875 \\
& FW10M & 7 & 6 & 9 & 7 \\
$\gamma=0.10, r=0.75$ & CC & 0.942 & 0.937 & 0.908 & 0.921 \\
& FW10M & 8 & 8 & 10 & 12 \\
MLEM & CC & 0.915 & 0.885 & 0.829 & 0.798 \\
& FW10M & 8 & 9 & 8 & 14 \\
\hline
\end{tabular}

Table 5.

Comparison of image quality metrics for images reconstructed from GATE data with varying number of views and constant scan time per view.

Figure 16 compares images reconstructed with the proposed algorithm at the parameter values that yielded the highest $\mathrm{CC}(\mathrm{Y}=$ $0.01, r=1.5$ and $Y=0.01, r=0.75$ ) and MLEM for a varying number of angular views ( 9 to 60 ) and a constant 10 second acquisition time for each angular position of the three-camera system. Thus the total scan time was 200, 70, 50, and 30 seconds for 60, 21, 15, and 9 views, respectively. Associated image quality metrics are presented in Table 5. As scan time and angular sampling decreased, images reconstructed using the proposed algorithm with $\mathrm{y}=0.01$ and $r=$ 1.50 showed decreased accuracy compared to scans with less noise and the same angular sampling presented in the previous section (Table 5 compared to Table 4). When reconstructing from 21 views, 15 views and 9 views, higher $\mathrm{CC}$ was achieved using $\mathrm{Y}=0.1$ and $r=$ 0.75 , compared to using $Y=0.01$ and $r=1.50$. In both cases, the proposed reconstruction algorithm provided higher CC than MLEM.

Physics in Medicine and Biology, Vol. 58, No. 16 (August 2013): pg. 5629-5652. DOI. This article is (C) Institute of Physics and permission has been granted for this version to appear in e-Publications@Marquette. Institute of Physics does not grant permission for this article to be further copied/distributed or hosted elsewhere without the express permission from Institute of Physics. 
NOT THE PUBLISHED VERSION; this is the author's final, peer-reviewed manuscript. The published version may be accessed by following the link in the citation at the bottom of the page.

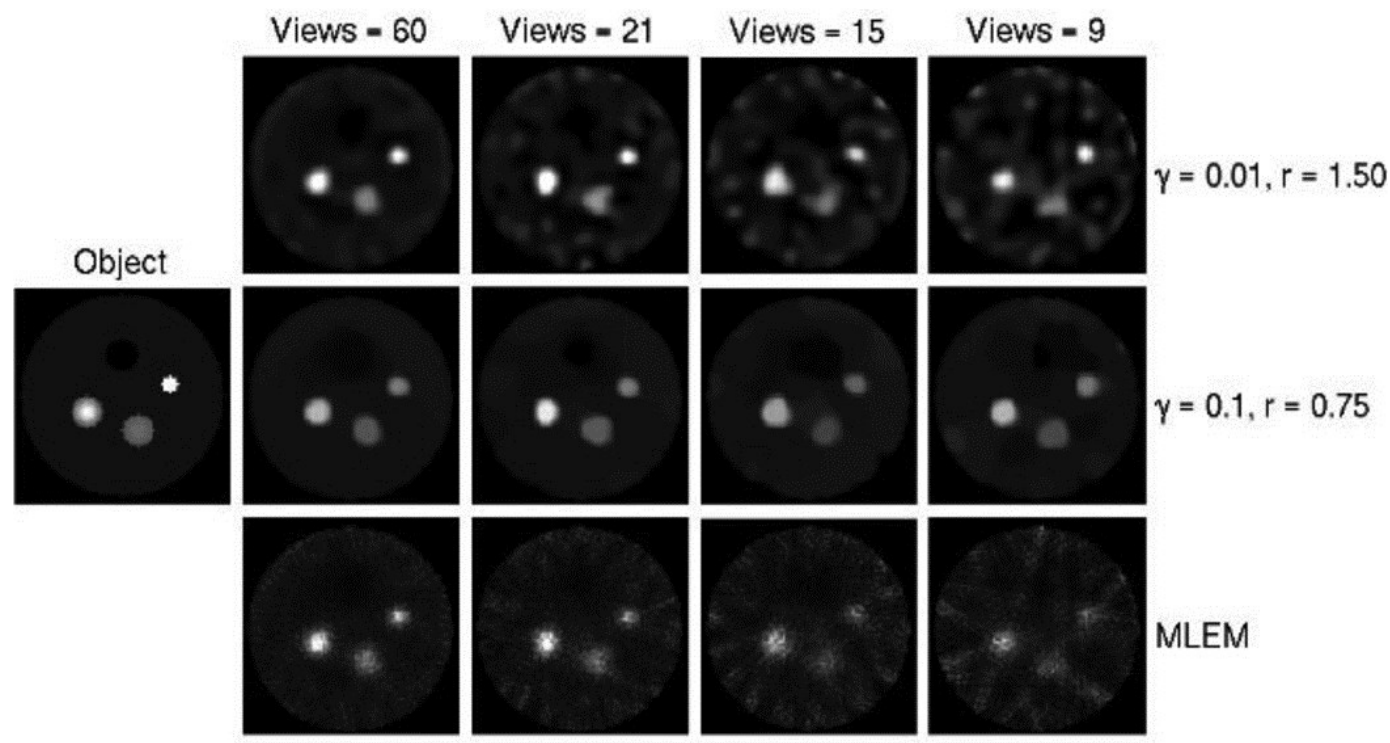

Figure 16.

Images reconstructed using the proposed algorithm and MLEM from GATE data simulated with the same time per view for different numbers of views.

\section{Monte Carlo simulation study with cardiac phantom}

This study examined the reconstruction of a phantom with more realistic physiological structure. Projection data were generated using Monte Carlo simulations as described in section 4.

\subsection{Methods}

\subsubsection{Phantom}

The phantom in this study was adapted from a 4D XCAT phantom (Segars et al 2010). A single slice of the phantom was simulated and assumed to be static. The field of view included the myocardium with a septal defect. The phantom was defined on a 128 $\times 128$ pixel grid as shown in figure 17, and loaded into GATE as one slice of a voxelized phantom. The voxel dimensions of the simulated phantom were set to $0.2 \mathrm{~mm}$ so that the phantom was contained within the field of view of the three-camera SPECT system described in figure 9 and table 3 . The reconstructed pixel size was $0.5 \mathrm{~mm} \times 0.5$ $\mathrm{mm}$.

Physics in Medicine and Biology, Vol. 58, No. 16 (August 2013): pg. 5629-5652. DOI. This article is (C) Institute of Physics and permission has been granted for this version to appear in e-Publications@Marquette. Institute of Physics does not grant permission for this article to be further copied/distributed or hosted elsewhere without the express permission from Institute of Physics. 


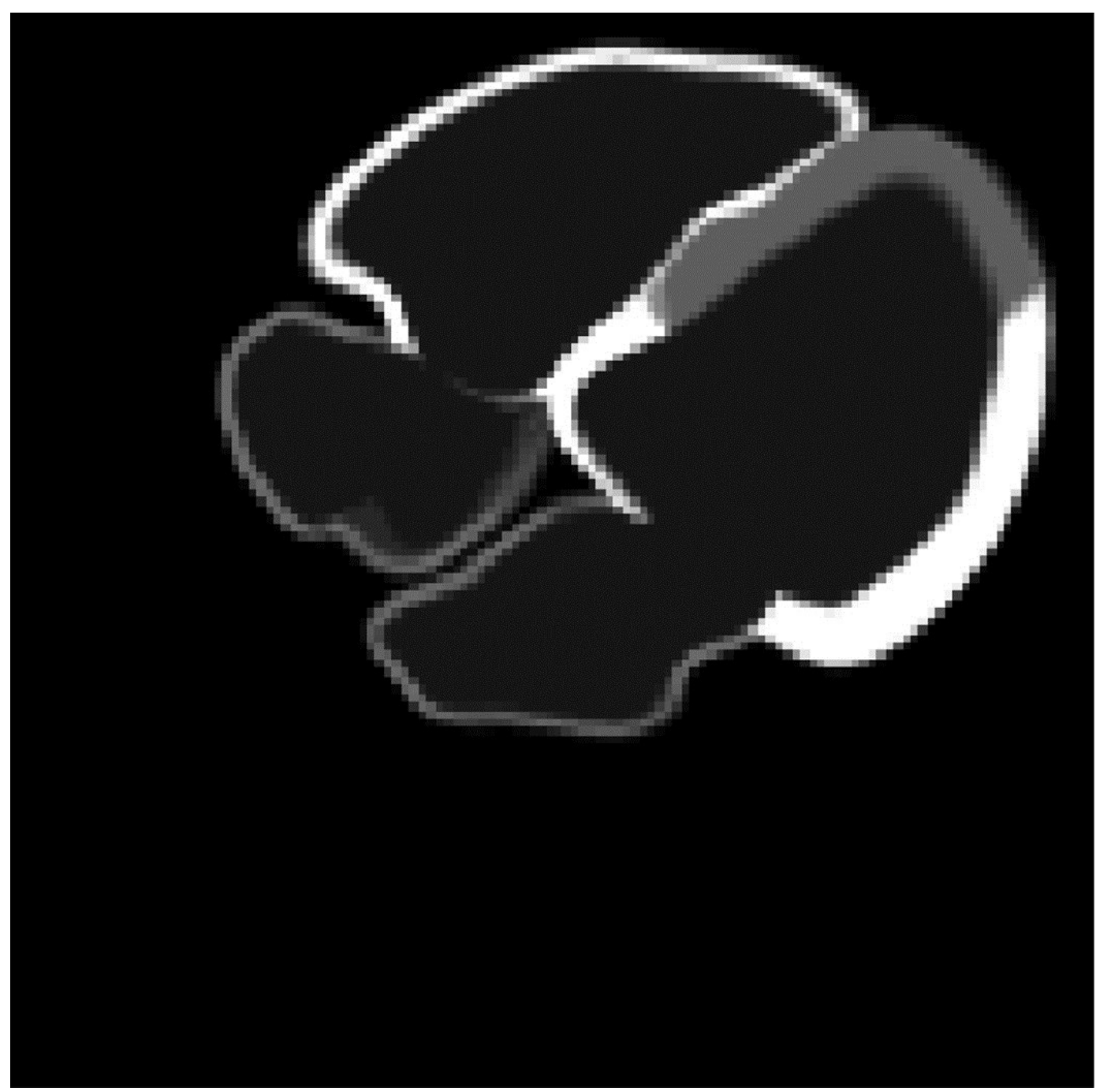

Figure 17.

Voxelized phantom adapted from XCAT phantom used in this study.

\section{Simulation}

Projections of the voxelized cardiac phantom were generated with Monte Carlo simulations modeling the three-camera system described in section 4.1.2. Acquisitions were simulated with 60 views, 15 views and 9 views modeling approximately 50,000 total detected counts over a total 200 second acquisition time. This total number of detected counts was selected to be similar to the phantom in Section 4 , whose peak activity levels were selected to be within the ranges of those measured in a previous rat lung study (Ma et al 2013). 
Reconstructions were performed with $y=0.01, r=1.5$ and $y=0.1, r$ $=0.75$, as these parameters were found to provide the highest $C \mathrm{C}$ in the study described in Section 4. Reconstructed image quality was evaluated using the CC metric described previously and compared to MLEM reconstructions. The phantom was resampled to a grid of reconstruction pixels $(0.5 \mathrm{~mm} \times 0.5 \mathrm{~mm})$ and used as the "true" image in the CC calculation.

\subsection{Results}

Reconstructed images are shown in figure 18 with table 6 listing the value of the $\mathrm{CC}$ metric for each image. Images reconstructed using $\mathrm{Y}=0.01, r=1.5$ resulted in the highest CC values, with a $2.7 \%$ decrease in CC as the number of views decreases from 60 views to nine views. This set of reconstruction parameters resulted in some patchy artifacts in the ventricular region, which were reduced using $Y$ $=0.1, r=0.75$. As $\mathrm{y}$ is increased from 0.01 to 0.1 , the reconstructed images are subject to greater regularization (because of the selection of $\mathrm{Y}$ ), resulting in a $1 \%$ decrease in $\mathrm{CC}$ as the number views decreased from 60 to nine, but lower overall CC. The proposed reconstruction algorithm provided images with higher CC compared to MLEM. The CC values for all three reconstruction methods were lower than those measured in images of the contrast element phantom (section 4), including images reconstructed from 60 views, likely due to limitations in the spatial resolution of the system for depicting the fine phantom structures. 
NOT THE PUBLISHED VERSION; this is the author's final, peer-reviewed manuscript. The published version may be accessed by following the link in the citation at the bottom of the page.

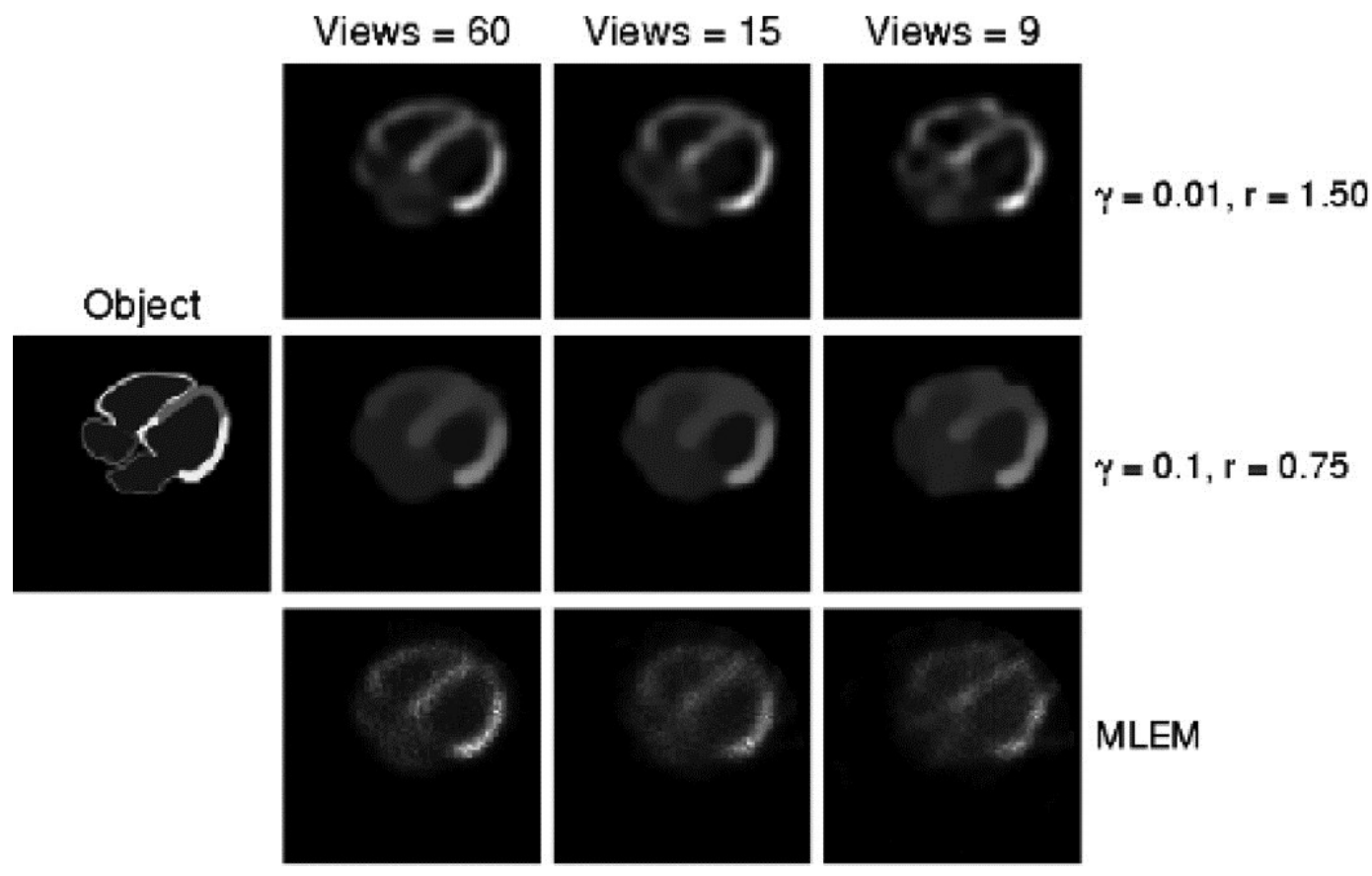

Figure 18.

Images reconstructed from 60 views, 15 views and 9 views using the proposed algorithm and MLEM. Data were generated from GATE simulation of a cardiac phantom. MLEM reconstructions are presented as a reference. As in previous sections, the MLEM stopping iteration was selected as that with the highest CC value.

\begin{tabular}{llll}
\hline & 60 views & 15 views & 9 views \\
\hline$\gamma=0.01, r=1.50$ & 0.782 & 0.772 & 0.760 \\
$\gamma=0.10, r=0.75$ & 0.757 & 0.753 & 0.750 \\
MLEM & 0.750 & 0.733 & 0.717 \\
\hline
\end{tabular}

Table 6.

Comparison of CC metric for reconstructions using different reconstruction protocols.

\section{Discussion}

The studies in which data were generated using the system matrix and blurring model indicated that accurate reconstruction may be possible when the incorrect blurring model is used, as there was only a $2.5 \%$ decrease in the CC metric over all $r$ studied when $y=0.1$ and $Y=0.01$. However, in the few-view case, using an $r$ larger than $r_{\text {true }}$ caused the number of meaningful coefficients in the intermediate image $f$ to increase rapidly compared to using lower values of $r$. This indicates a less sparse image, limiting the effectiveness of exploiting

Physics in Medicine and Biology, Vol. 58, No. 16 (August 2013): pg. 5629-5652. DOI. This article is (C) Institute of Physics and permission has been granted for this version to appear in e-Publications@Marquette. Institute of Physics does not grant permission for this article to be further copied/distributed or hosted elsewhere without the express permission from Institute of Physics. 
gradient-magnitude sparsity to reduce the number of views needed for reconstruction. When an approximately accurate blurring model is used, the intermediate image $f$ is the most sparse in the gradientmagnitude sense. This may allow a greater reduction in the sampling necessary for reconstruction.

The results demonstrate that blurring and noise regularization increased with increasing values of $r$, the standard deviation of the Gaussian blurring kernel, and $\mathrm{Y}$, the TV weighting parameter. For example, in the high-view case with noiseless data generated from the system model (Section 3), reconstructions using the lowest $\mathrm{y}$ value studied ( $\gamma=0.0001$ ) yielded the most accurate images for a given value of $r$. When the data were made inconsistent by the addition of Poisson noise, the optimal studied $y$ value increased to $Y=0.01$.

In the GATE simulation studies of the contrast element phantom (Section 4)and cardiac phantom (Section 5), $\mathrm{Y}=0.01, r=1.5$ and $\mathrm{Y}=$ $0.1, r=0.75$ yielded higher CC compared to MLEM reconstructions, even though the object was not exactly represented by the blurring model assumed by the reconstruction algorithm. Parameters $Y=0.01$, $r=1.5$ resulted in the highest $\mathrm{CC}$ when the view sampling was decreased with total number of counts held constant. When the number of counts decreased with the view sampling, parameters $Y=$ $0.1, r=0.75$ resulted in the highest CC at low views. The two studied parameter settings resulted in similar CC but different qualitative attributes (figures 14, 16,16, and and18).18). Overall, reconstructions from data generated using GATE simulations suggest that when the true blurring model is unknown and noise is present, lower values of $Y$ $(\mathrm{Y}=0.01$ in this particular study) benefit from larger $r$ values, while $\mathrm{Y}$ $=0.1$ benefits from lower $r$ values, with a smaller dependence on $r$. Since the inverse crime study demonstrated that smaller $r$ values result in a more sparse intermediate image, the combination of $\mathrm{Y}=$ 0.1 and $r=0.75$ may be advantageous for reconstruction from fewviews. The preferred parameter combination requires further study with human or model observers to quantify the effects of the patchy artifacts.

The results also suggest that, when an appropriate value of the TV penalty term is included in the proposed reconstruction algorithm $(Y=0.01$ or 0.1 for the cases studied $)$, streaking artifacts are reduced

Physics in Medicine and Biology, Vol. 58, No. 16 (August 2013): pg. 5629-5652. DOI. This article is (C) Institute of Physics and permission has been granted for this version to appear in e-Publications@Marquette. Institute of Physics does not grant permission for this article to be further copied/distributed or hosted elsewhere without the express permission from Institute of Physics. 
compared to MLEM reconstructions. Images reconstructed with the proposed algorithm contain low frequency variations (patchy artifacts) in high-noise simulation cases (figures 14 and and16).16). Low frequency, patchy artifacts have been noted in CT TV reconstructions from noisy data, and future work is required to quantify the impact of these artifacts on the ability of observers to identify objects of diagnostic interest (Tang et al 2009).

The presented work suggests potential benefits of the proposed reconstruction algorithm compared to MLEM, however, additional work is required for a systematic comparison, including experimental investigation. The algorithm is expected to have the most benefit for objects whose distribution is similar to the phenomenological blurred piecewise constant model (i.e., distributions for which the activity may have constant regions, but varies smoothly between regions). Thus, the algorithm may be most beneficial for perfusion studies, as is supported by the results of the cardiac phantom. However, the cardiac phantom is only an approximation of the expected object, thus in-vivo studies are required for further evaluation. The simulation results suggest that the algorithm may be beneficial even when the object does not match the phenomenological model. In vivo experimental studies are required to quantify the algorithm performance for different applications.

One limitation of the presented work is that the simulations modeled 2D objects and acquisition, whereas SPECT data are acquired in three dimensions. The aim of this work is to solve the blurred TV model to high accuracy and to understand the contribution from optimization problem error. Future work will explore approximate model solution which will presumably be more computationally efficient and generalizable to three dimensions. We hypothesize that the principles and model presented in this work can be generalized to a 3D case with the expansion of the system matrix and applying the blurring-masking function in three dimensions. Additional studies are necessary to investigate this hypothesis. Reconstruction from multipinhole systems could be accomplished by modifying the system matrix to include contributions from all pinholes. This study focused on small-animal pinhole SPECT systems, as stationary systems have been proposed with few view acquisition for dynamic imaging (Beekman et al 2005, Furenlid et al 2004, Beekman and Vastenhouw 2004). The and permission has been granted for this version to appear in e-Publications@Marquette. Institute of Physics does not grant permission for this article to be further copied/distributed or hosted elsewhere without the express permission from Institute of Physics. 
proposed algorithm can be used with arbitrary system geometries e.g. parallel hole collimators, focusing collimators, provided that the system matrix appropriately models the system and its transpose can be found. Modifications to the system matrix (3D, multiple pinhole, and different collimators) represent changes in ray sampling. We hypothesize that the results presented here can be reasonably extrapolated to different scanning geometries with similar information content, as iterative reconstruction methods are typically less sensitive to sampling than filtered backprojection approaches. Future work will investigate the performance of this algorithm for dynamic imaging from few-views.

\section{Conclusions}

This study proposed and characterized a sparsity-exploiting reconstruction algorithm for SPECT that is intended for few-view imaging and that phenomenologically models the object as piecewise constant subject to a blurring operation. While the reconstruction technique assumes a specific blurring model, the results demonstrate that the knowledge of the true blurring parameter is not required for accurate reconstruction, as the reconstruction algorithm has limited sensitivity to $r$ in the low noise cases and benefits from increasing $r$ in the high noise case. However, the results suggest that accurately modeling the blurring parameter provides increased gradientmagnitude sparsity, which may enable further reductions in sampling. The reconstructed images demonstrate that the algorithm introduces low-frequency artifacts in the presence of noise, but eliminates streak artifacts due to angular undersampling. The effects of these artifacts on observers will be studied in future work. Overall, the results demonstrate preliminary feasibility of a sparsity-exploiting reconstruction algorithm which may be beneficial for few-view SPECT.

Listing 1: Pseudocode of the proposed algorithm

$$
\begin{aligned}
& L:=\|(\boldsymbol{H} \boldsymbol{M} \boldsymbol{G M}, \boldsymbol{D})\|_{2} ; \tau=\sigma=0.9 / L ; \theta=1 ; n=0 \\
& f_{0}:=f_{0}^{\prime}:=p_{0}:=\boldsymbol{q}_{0}:=0
\end{aligned}
$$




\section{Repeat}

$$
\begin{aligned}
& \boldsymbol{p}_{n+1}:=0.5\left(1+\boldsymbol{p}_{n}+\sigma \boldsymbol{H} \boldsymbol{M} \boldsymbol{G} \boldsymbol{M} \boldsymbol{f}_{n}^{\prime}-\left(\left(p_{n}+\sigma \boldsymbol{H} \boldsymbol{M} \boldsymbol{G} \boldsymbol{M} \boldsymbol{f}_{n}^{\prime}-1\right)^{2}+4 \sigma \boldsymbol{g}\right)^{1 / 2}\right. \\
& \boldsymbol{q}_{n+1}:=\gamma\left(\boldsymbol{q}_{n}+\sigma \boldsymbol{D} \boldsymbol{f}_{n}^{\prime}\right) / \max \left(\gamma,\left|\boldsymbol{q}_{n}+\sigma \boldsymbol{D} \boldsymbol{f}_{n}^{\prime}\right|\right) \\
& \boldsymbol{f}_{n+1}:=\boldsymbol{f}_{n}-\tau \boldsymbol{M} \boldsymbol{G} \boldsymbol{M} \boldsymbol{H}^{\mathrm{T}} \boldsymbol{p}_{n+1}+\tau \operatorname{div}\left(\boldsymbol{q}_{n+1}\right) \\
& \boldsymbol{f}_{n+1}^{\prime}:=\boldsymbol{f}_{n+1}+\theta\left(\boldsymbol{f}_{n+1}-\boldsymbol{f}_{n}\right) \\
& n=n+1
\end{aligned}
$$

Until stopping criterion

\section{Acknowledgments}

This work was supported in part by NIH R15 grant CA143713 and R01 grants CA158446, CA120540 and EB000225. The contents of this paper are solely the responsibility of the authors and do not necessarily represent the official views of the National Institutes of Health. This work is part of the project CSI: Computational Science in Imaging, supported by grant 274-07-0065 from the Danish Research Council for Technology and Production Sciences. The high performance computing resources used in this paper were funded by NSF grant OCI-0923037.

\section{References}

Barrett $\mathrm{HH}$, Myers KJ. In: Foundations of Image Science. Saleh EA, Bahaa, editors. Hoboken, NJ: John Wiley \& Sons, Inc; 2004.

Beekman FJ, van der Have F, Vastenhouw B, van der Linden AJa, van Rijk PP, Burbach JPH, Smidt MP. U-SPECT-I: a novel system for submillimeter-resolution tomography with radiolabeled molecules in mice. J. Nucl. Med. 2005;46:1194-1200.

Beekman FJ, Vastenhouw B. Design and simulation of a high-resolution stationary SPECT system for small animals. Phys. Med. Biol. 2004;49:4579-4592.

Chambolle A, Pock T. A first-order primal-dual algorithm for convex problems with applications to imaging. J. Math. Imag. Vis. 2011;40:1-26. 
NOT THE PUBLISHED VERSION; this is the author's final, peer-reviewed manuscript. The published version may be accessed by following the link in the citation at the bottom of the page.

Chen G-H, Tang J, Leng S. Prior image constrained compressed sensing (PICCS): A method to accurately reconstruct dynamic CT images from highly undersampled projection data sets. Med. Phys. 2008;35:660-663.

Donato P, Coelho P, Santos C, Bernardes A, Caseiro-Alves F. Correspondence between left ventricular 17 myocardial segments and coronary anatomy obtained by multi-detector computed tomography: an ex vivo contribution. Surgical and Radiologic Anatomy. 2012;34:805-810.

Duan X, Zhang L, Xing Y, Chen Z, Cheng J. Few-View Projection Reconstruction With an Iterative Reconstruction-Reprojection Algorithm and TV Constraint. IEEE Trans. Nucl. Sci. 2009;56:1377-1382.

Furenlid LR, Wilson DW, Chen Y-C, Kim H, Pietraski PJ, Crawford MJ, Barrett HH. FastSPECT II: A Second-Generation High-Resolution Dynamic SPECT Imager. IEEE Trans. Nucl. Sci. 2004;51:631635.

Gullberg GT. Dynamic SPECT imaging: exploring a new frontier in medical imaging. IEEE International Symposium on Biomedical Imaging: Nano to Macro. 2004:607-610.

Gullberg GT, Reutter BW, Sitek A, Maltz JS, Budinger TF. Dynamic single photon emission computed tomography--basic principles and cardiac applications. Phys. Med. Biol. 2010;55:R111-R191.

Hill DL, Batchelor PG, Holden M, Hawkes DJ. Medical image registration. Phys. Med. Biol. 2001;46:R1-R45.

Hudson $\mathrm{H}$, Larkin R. Accelerated image reconstruction using ordered subsets of projection data. IEEE Trans. Med. Img. 1994; 13:601-609.

Hsieh Hohui, Ingtsung Hsiao. "Image reconstructions from limit views and angle coverage data for a stationary multi-pinhole SPECT system". Tsinghua Science \& Technology. 2010;15:44-49.

Physics in Medicine and Biology, Vol. 58, No. 16 (August 2013): pg. 5629-5652. DOI. This article is (C) Institute of Physics and permission has been granted for this version to appear in e-Publications@Marquette. Institute of Physics does not grant permission for this article to be further copied/distributed or hosted elsewhere without the express permission from Institute of Physics. 
Jain R. Determinants of tumor blood flow: a review. Cancer Research. $1988 ; 48: 2641-2658$.

Jan S, Santin G, Strul D, Staelens S. GATE: a simulation toolkit for PET and SPECT. Phys. Med. Biol. 2004;49:4543.

Kaipio J, Somersalo E. In: Statistical and Computational Inverse Problems. Antman SS, Marsden JE, Sirovich L, editors. New York: Springer Science+Business Media, LLC; 2005.

Ma D, Wolf $P$, Clough A, Schmidt T. The Performance of MLEM for Dynamic Imaging From Simulated Few-View, Multi-Pinhole SPECT. IEEE Trans. Nucl. Sci. 2013;60:115-123.

Mukherjee, Joyeeta $M$, et al. "Objective assessment of penalized maximum likelihood reconstruction with sparsity-promoting penalty for Myocardial perfusion SPECT imaging.". SPIE Medical Imaging. International Society for Optics and Photonics. 2013

Pereztol-Valdés O, Candell-Riera J, Santana-Boado C, Angel J, AguadéBruix S, Castell-Conesa J, Garcia EV, Soler-Soler J. Correspondence between left ventricular 17 myocardial segments and coronary arteries. European Heart Journal. 2005;26:2637-2643.

Ritschl L, Bergner F, Fleischmann C, Kachelriess M. Improved total variation-based $C T$ image reconstruction applied to clinical data. Phys. Med. Biol. 2011;56:1545-1561.

Segars WP, Sturgeon G, Mendonca S, Grimes J, Tsui BMW. 4D XCAT phantom for multimodality imaging research. Med. Phys. 2010;37:4902-4915.

Shepp LA, Vardi Y. Maximum likelihood reconstruction for emission tomography. IEEE Trans. Med. Img. 1982;1:113-122.

Siddon RL. Fast calculation of the exact radiological path for a threedimensional CT array. Med. Phys. 1985;12:252-255.

Physics in Medicine and Biology, Vol. 58, No. 16 (August 2013): pg. 5629-5652. DOI. This article is (C) Institute of Physics and permission has been granted for this version to appear in e-Publications@Marquette. Institute of Physics does not grant permission for this article to be further copied/distributed or hosted elsewhere without the express permission from Institute of Physics. 
NOT THE PUBLISHED VERSION; this is the author's final, peer-reviewed manuscript. The published version may be accessed by following the link in the citation at the bottom of the page.

Sidky E, Kao C, Pan X. Accurate image reconstruction from few-views and limited-angle data in divergent-beam CT. J. X-ray Sci. Tech. 2006;14:119-139.

Sidky EY, Jørgensen JH, Pan X. Convex optimization problem prototyping for image reconstruction in computed tomography with the Chambolle-Pock algorithm. Phys. Med. Biol. 2012;57:3065-3091.

Sidky EY, Pan X. Image reconstruction in circular cone-beam computed tomography by constrained, total-variation minimization. Phys. Med. Biol. 2008;53:4777-4807.

Sidky EY, Pan X, Reiser IS, Nishikawa RM, Moore RH, Kopans DB. Enhanced imaging of microcalcifications in digital breast tomosynthesis through improved image-reconstruction algorithms. Med. Phys. 2009;36:4920-4932.

Tang J, Nett BE, Chen G-H. Performance comparison between total variation (TV)-based compressed sensing and statistical iterative reconstruction algorithms. Phys. Med. Biol. 2009;54:57815804.

Vandenberghe S, D'Asseler Y, Van de Walle R, Kauppinen T, Koole M, Bouwens L, Van Laere K, Lemahieu I, Dierckx Ra. Iterative reconstruction algorithms in nuclear medicine. Comput. Med. Imaging Graphics. 2001;25:105-111.

Vanhove C, Defrise M, Lahoutte T, Bossuyt A. Three-pinhole collimator to improve axial spatial resolution and sensitivity in pinhole SPECT. Eur. J. Nucl. Med. Mol. Imaging. 2008;35:407-415.

Physics in Medicine and Biology, Vol. 58, No. 16 (August 2013): pg. 5629-5652. DOI. This article is (C) Institute of Physics and permission has been granted for this version to appear in e-Publications@Marquette. Institute of Physics does not grant permission for this article to be further copied/distributed or hosted elsewhere without the express permission from Institute of Physics. 
NOT THE PUBLISHED VERSION; this is the author's final, peer-reviewed manuscript. The published version may be accessed by following the link in the citation at the bottom of the page.

\section{About the Authors:}

${ }^{1}$ Department of Biomedical Engineering, Marquette University, 1515 W. Wisconsin Ave. Milwaukee, WI 53233, USA

${ }^{2}$ Department of Applied Mathematics and Computer Science, Technical University of Denmark, Matematiktorvet, Building 303B, 2800 Kgs. Lyngby, Denmark

${ }^{3}$ Department of Radiology, University of Chicago, 5841 S. Maryland Ave., Chicago, IL 60637, USA

Physics in Medicine and Biology, Vol. 58, No. 16 (August 2013): pg. 5629-5652. DOI. This article is (C) Institute of Physics and permission has been granted for this version to appear in e-Publications@Marquette. Institute of Physics does not grant permission for this article to be further copied/distributed or hosted elsewhere without the express permission from Institute of Physics. 